\title{
THE COMPACT STAR-FORMING COMPLEX AT THE HEART OF NGC $253^{1,2,3}$
}

\author{
T. J. Davidge \\ Dominion Astrophysical Observatory, \\ National Research Council of Canada, 5071 West Saanich Road, \\ Victoria, BC Canada V9E 2EY \\ tim.davidge@nrc.ca
}

\begin{abstract}
We discuss integral field spectra of the compact star-forming complex that is the brightest near-infrared (NIR) source in the central regions of the starburst galaxy NGC 253. The spectra cover the $H$ and $K$ passbands and were recorded with the Gemini NIR Spectrograph during sub-arcsec seeing conditions. Absorption features in the spectrum of the star-forming complex are weaker than in the surroundings. An absorption feature is found near $1.78 \mu \mathrm{m}$ that coincides with the location of a $\mathrm{C}_{2}$ bandhead. If this feature is due to $\mathrm{C}_{2}$ then the star-forming complex has been in place for at least a few hundred Myr. Emission lines of Br $\gamma$, [FeII], and HeI2.06 $\mu \mathrm{m}$ do not track the NIR continuum light. Pockets of starforming activity that do not have associated concentrations of red supergiants, and so likely have ages $<8 \mathrm{Myr}$, are found along the western edge of the complex, and there is evidence that one such pocket contains a rich population of WolfRayet stars. Unless the star-forming complex is significantly more metal-poor than the surroundings, then a significant fraction of its total mass is in stars with
\end{abstract}

\footnotetext{
${ }^{1}$ Based on observations obtained at the Gemini Observatory, which is operated by the Association of Universities for Research in Astronomy, Inc., under a cooperative agreement with the NSF on behalf of the Gemini partnership: the National Science Foundation (United States), the National Research Council (Canada), CONICYT (Chile), the Australian Research Council (Australia), Ministério da Ciência, Tecnologia e Inovação (Brazil) and Ministerio de Ciencia, Tecnología e Innovación Productiva (Argentina).

${ }^{2}$ This research used the facilities of the Canadian Astronomy Data Centre operated by the National Research Council of Canada with the support of the Canadian Space Agency.

${ }^{3}$ This research has made use of the NASA/IPAC Infrared Science Archive, which is operated by the Jet Propulsion Laboratory, California Institute of Technology, under contract with the National Aeronautics and Space Administration.
} 
ages $<8$ Myr. If the present-day star formation rate is maintained then the time scale to double its stellar mass ranges from a few Myr to a few tens of Myr, depending on the contribution made by stars older than $\sim 8 \mathrm{Myr}$. If - as suggested by some studies - the star-forming complex is centered on the galaxy nucleus, which presumably contains a large population of old and intermediate-age stars, then the nucleus of NGC 253 is currently experiencing a phase of rapid growth in its stellar mass.

Subject headings: galaxies: individual (NGC 253) - galaxies: starburst - galaxies: spiral - galaxies: star clusters: general - galaxies: evolution

\section{INTRODUCTION}

Interactions and mergers can spur the large-scale re-distribution of material and angular momentum within a galaxy, thereby altering basic observational properties such as morphology. Some of the material that is displaced by such events will be channeled into the central regions of galaxies, where elevated levels of star-forming activity will be triggered in concert with those at large radii. Observations suggest that while elevated episodes of star-forming activity in a galaxy may die out first at large radii, it may continue for some time in the central regions (e.g. Soto \& Martin 2010). Given that the typical damping time for star burst activity in moderately low mass galaxies is many hundreds of Myr (McQuinn

et al. 2010), then we might expect to find evidence for extensive star-forming activity in the central regions of late stage starbursts that is spread over a broad time period.

The barred spiral galaxy NGC 253 is the dominant member of one of the three concentrations that collectively make up the Sculptor Group (Karachentsev et al. 2003). NGC 253 is experiencing a starburst (e.g. Rieke et al. 1980), and is one of the nearest such examples of this phenomenon. NGC 253 does not have an entourage of bright companions, and it is not clear what event (or events) triggered the starburst. Based on a survey of bright evolved stars in the NGC 253 disk, Davidge (2010) suggests that NGC 253 interacted with 
a now defunct companion within the past 0.2 Gyr. Such an encounter could have spurred the formation of the bar, and caused the re-distribution of gas and dust throughout the galaxy (e.g. Hopkins et al. 2009). Alternatively, a flyby encounter with another large galaxy could also have triggered bar formation and starburst activity (Lang et al. 2014). If a flyby happened more than $\sim 100 \mathrm{Myr}$ in the past then identifying the perturbing galaxy may be problematic.

The central regions of NGC 253 contain clues about the age and starburst triggering mechanism. The center of NGC 253 is heavily obscured at visible wavelengths, and so most previous studies have been conducted in the near-infrared (NIR). Engelbracht et al. (1998) used long-slit spectra and narrow-band images to investigate the ionization properties, stellar content and structural characteristics in a 7.5 arcsec radius aperture that contains most of the [NeII], $\mathrm{Br} \gamma, \mathrm{H}_{2}$, and mid-infrared (MIR) flux. They conclude that the interstellar medium (ISM) is excited by a radiation field that is powered by objects with $\mathrm{T}_{\text {eff }} \leq 38000 \mathrm{~K}$, indicating that a large population of young stars is present. This young component formed within the past $\sim 30 \mathrm{Myr}$ and contributes $\sim 10-20 \%$ of the stellar mass in the region studied.

There is a compact bright structure that defines the photometric center of NGC 253 in the NIR. This object, which has a sub-arcsec characteristic width, and its immediate surroundings are the subject of this paper. Following Gunthardt et al. (2015), we refer to this object as the IR Core (hereafter IRC). Modelling of $\operatorname{Br} \gamma$ emission by Kornei \& McCrady (2009) suggests that the IRC contains a large number of ionizing stars that have an age of 5.7 Myr, and they estimate that its total stellar mass is $1.4 \times 10^{7} \mathrm{M}_{\odot}$. Kornei \& McCrady (2009) further note that the IRC has been the site of an extended period of star-forming activity during recent epochs. This conclusion is based on the deep $\mathrm{CO}$ absorption bands that are seen in the integrated spectrum at $\lambda>2.3 \mu \mathrm{m}$. Deep CO bands in the integrated spectra of young clusters originate in red supergiants (RSGs), and so are signatures of systems with 
ages older than $\sim 8-10$ Myr.

The IRC is offset by a few arcsec from what has been identified as the kinematic center of the galaxy (Muller-Sanchez et al. 2010; Rosenberg et al. 2013). HOwever, Rosenberg et al. (2013) also find differences between the distributions of NIR continuum light, $\operatorname{Br} \gamma$ emission, and $\mathrm{H}_{2}$ emission, indicating that the stellar content near the center of NGC 253 has complex spatial and kinematic properties. Additional evidence of complexity is presented by Gunthardt et al. (2015), who find sub-structuring and a complicated projected distribution of stellar types in and around the IRC. They find that the IRC coincides with the rotational center of $\mathrm{H}_{2}$ emission, and argue that the IRC is the true nucleus of NGC 253.

The complex projected distribution of stars and gas, coupled with the evidence for sub-structuring, demonstrates the need to map the distribution of spectroscopic features in and around the IRC. This task is well-suited to spectrographs that are equipped with an integral field unit (IFU). In the present study, deep IFU spectra of the central regions of NGC 253 that cover the $1.4-2.3 \mu \mathrm{m}$ wavelength interval are discussed. This wavelength interval contains absorption and emission features that can be used to trace the projected distribution of stars that span a wide range of ages, and identify areas of recent star-forming activity.

The nucleus of the late-type disk galaxy NGC 7793, which is the dominant member of another Sculptor Group concentration (Karachensheva et al. 2003), was also observed to represent the nucleus of a non-bursting, isolated spiral galaxy. To the best of our knowledge, these are the first NIR spectra of the central regions of NGC 7793. Bibby \& Crowther (2010) estimate from the $\mathrm{H} \alpha$ flux that the $\mathrm{SFR}$ in $\mathrm{NGC} 7793$ is $0.45 \mathrm{M}_{\odot}$ year ${ }^{-1}$, and this is $\sim 6 \times$ lower than the SFR of NGC 253 measured by Ott et al. (2005). Carson et al. (2015) find that the photometric size of the NGC 7793 nucleus grows with decreasing wavelength, as expected if there is a spatially extended young population. 
Unlike NGC 253, there are indications that NGC 7793 has evolved in isolation throughout much of its life. The disk of NGC 7793 spans many scale lengths (Vlajic et al. 2011), and Radburn-Smith et al. (2012) suggest that the outermost regions of its disk were populated by radial migration from smaller radii, rather than by the disruption of an earlier structure. Davidge (1998) found that the NGC 7793 disk contains stars that span a broad range of ages, and the oldest of these have an age $\sim 10$ Gyr. The age and radial extent of the NGC 7793 disk are thus consistent with it having been in place for a long period of time and not having been disrupted by a merger or interaction.

Details of the observations and the reduction of the data can be found in Section 2 . Archival NIR images that provide supplemental information for interpreting the spectra are discussed in Section 3. The strengths and on-sky distributions of spectroscopic features that are used to probe stellar content are discussed in Sections 4 and 5. Comparisons are made with model spectra in Section 6. A summary and discussion of the results are presented in Section 7.

\section{OBSERVATIONS \& REDUCTION}

The spectra were recorded at Gemini South (GS) on the night of UT November 21, 2004 UT with the Gemini NIR Spectrograph (GNIRS; Elias et al. 2006) as part of program GS2004B-SV-22 (PI: Davidge). The GNIRS IFU is described in detail by Dubbeldam et al. (2000). The IFU was 1 an image slicer design with 21 slitlets. It was used in low resolution mode for these observations, and so a $3.15 \times 4.85$ arcsec field was covered on the sky. Each slitlet is 0.15 arcsec wide and subtends 32 pixels on the $1024 \times 1024 \mathrm{InSb}$ array in GNIRS, so that the sampling along the slit is 0.15 arcsec pixel $^{-1}$. Each spaxal thus subtends $0.15 \times 0.15$ arcsec.

\footnotetext{
${ }^{1}$ The IFU was destroyed during a run-away instrument warm-up in 2007.
} 
The IRC was positioned at the center of the IFU science field. While there are no point sources in the IFU observations from which the image quality can be measured directly, the IRC has a FWHM $\sim 0.6-0.7$ arcsec in the $K$-band continuum wavelength interval defined in Section 5.1. Given that the IRC is an extended object, then this sets an upper limit to the size of the seeing disk.

A complete observation consisted of (1) five exposures of the target galaxy, with each exposure recorded at slightly different dither positions to assist with the identification and suppression of bad pixels and cosmic rays, followed by (2) five observations of a background sky field recorded with the same exposure times as the galaxy observations. This galaxy+sky sequence was repeated three times in each filter. Individual exposure times were 20 seconds for NGC 253 and 40 seconds for NGC 7793. The spectra presented here thus have total on-source integration times per filter of $5 \times 3 \times 20$ seconds $=300$ seconds for NGC 253, and $5 \times 3 \times 40$ seconds $=600$ seconds for NGC 7793.

Ancillary data were recorded to monitor instrumental and atmospheric characteristics. An Ar lamp spectrum was recorded after each galaxy+sky pair, while a series of flat-field frames were recorded at various times during the night, with the slit illuminated by a light source in the Gemini Facility Calibration Unit. Telluric absorption features were monitored with spectra of the F4V star HR8999, which was observed at different times throughout the night. Hydrogen absorption lines in the HR8999 spectrum were removed by subtracting Voigt profiles that were fitted to those lines.

The first step in the data reduction sequence was the removal of sky emission. A sky emission template was constructed for each galaxy spectrum by interpolating in time between the sky observations, and the result was subtracted from the spectrum. The skysubtracted frames in a given filter at each dither position were then co-added, and the results were divided by a flat field frame to correct for pixel-to-pixel sensitivity variations and non-uniformities in optical throughput. 
The mean flat-fielded exposures were aligned by centering on the peak signal in each slitlet, and the results were combined by averaging the signal at each shifted pixel location. This was followed by wavelength calibration, which was done using a dispersion solution obtained from the arc spectra. The wavelength-calibrated galaxy spectra were then divided by the telluric star spectrum.

\section{NARROW-BAND IMAGING}

The central regions of NGC 253 have a complex morphology, and even a single pointing with the GNIRS IFU samples a number of different structures. Moderately deep archival images of the center of NGC 253 were thus examined in an effort to better understand the region near the IRC. F128N and F164N WFC3 images that were recorded by the Hubble Space Telescope for program 12206 (PI: Westmoquette) and that had been processed with the WFC3 pipeline were downloaded from the Canadian Astronomical Data Center 2. While a combination of broad and narrow-band images were recorded for this program, the central regions of NGC 253 are saturated in the broad-band images, and so only the narrow-band data are considered here.

Three images were recorded in each filter, and these were aligned and median-combined on a filter-by-filter basis. The left hand panel of Figure 1 shows the central $6.7 \times 6.7$ arcsec of the stacked F164N image, with the IFU footprint indicated in black. The stacked F128N image was smoothed to match the angular resolution of the F164N image, and F128N-F164N colors are displayed in the right hand panel of Figure 1.

The F128N-F164N color varies throughout the area sampled with the IFU. A filament with moderately red color runs E/W through the IRC, and is flanked by two pockets with blue F128N-F164N colors. The detection of areas with very blue colors in the IRC is perhaps

\footnotetext{
${ }^{2}$ http://www.cadc-ccda.hia-iha.nrc-cnrc.gc.ca/en/hst/
} 


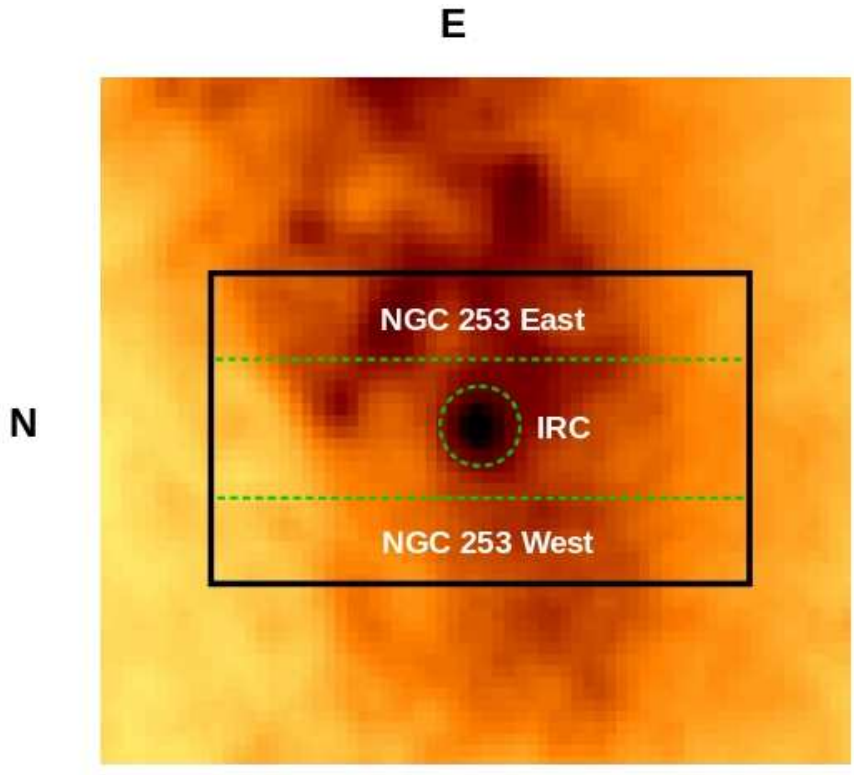

F164N

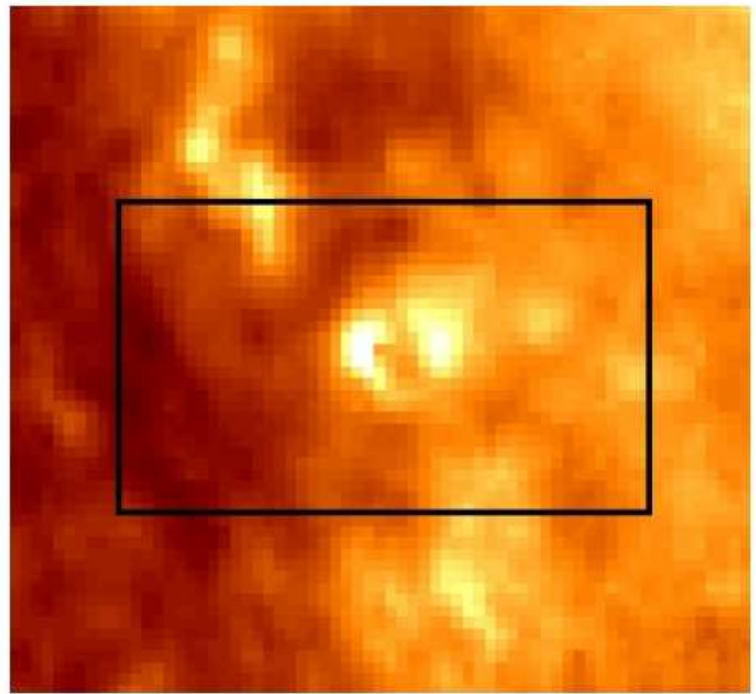

F128N-F164N

Fig. 1. - Narrow-band HST WFC3 images of the central $6.7 \times 6.7$ arcsec of NGC 253. The left hand panel shows the F164N image with North to the left and East at the top. The area sampled by the IFU is outlined in black, while the areas from which the IRC, NGC 253 East, and NGC 253 West spectra (Section 4) were extracted are shown in green. F128N-F164N color, displayed in magnitude units, is shown in the right hand panel, with redder colors having darker shading. There is a $\sim 0.9$ magnitude variation in F128N-F164N throughout the IFU field, and at least some of the structures in the F164N image are probably due to dust. 
not surprising given the spectroscopic evidence for very young stars there (Section 4). The red filament may be a dust lane, or a region between the blue pockets that is dominated by red stars. The filament has a color that is comparable to the area to the south of the IRC and is not as red as some of the suspected dust features, and this is consistent with the latter possibility. That the F128N-F164N color varies in the IRC foreshadows one of the general results of this paper, which is that the age distribution of stars in and around the IRC varies with location - the IRC is not a simple stellar population, but is instead a compact star-forming complex. Additional evidence to support this claim is presented in Section 5.

Aside from the IRC, other less prominent structures are also sampled by the IFU, with most of these to the east and north of the IRC. Kornei \& McCrady (2009) note the presence of dust lanes within a few arcsec of the IRC in their NIR images, and it is likely that some of the structures in Figure 1 are not physically distinct entities, but may instead be artifacts of dust absorption. The regions between the IRC and the knots that are $\sim 1$ arcsec to the north east of the IRC are separated by a filament that - owing to its very red color - is a

candidate dust lane. The north east corner of the IFU field contains an area with a blue F128N-F164N color, and in Section 5 it is demonstrated that this area shows spectroscopic signatures of very recent star formation. Finally, the north west corner of the IFU samples a region with red F128N-F164N colors, and a filamentary structure that appears to be a dust lane is also seen there in the F164N image.

\section{RESULTS: EXTRACTED SPECTRA}

Using the F164N image in Figure 1 as a guide, spectra that sample three different regions were extracted for detailed investigation: (1) the IRC, (2) the eastern portion of the IFU, where faint sub-structures are evident, and (3) the western portion of the IFU, where the light distribution is more uniform than elsewhere in the area sampled. The IRC spectrum 
was constructed by summing signal within a 0.4 arcsec radius circular gather centered on the IRC, which is where the signal was at least one half of the peak value. The other two spectra were obtained by combining signal in the $0.8 \times 4.85$ arcsec areas at the eastern and western edges of the IFU. These regions thus contain signal from sources that are at least 0.8 arcsec from the center of the IRC, and so contamination from the IRC should not be a concern. The extraction regions are indicated in Figure 1. The spatial distributions of prominent spectroscopic features at finer angular resolutions are examined in Section 5, while the extracted spectra are compared with model spectra in Section 6.

\subsection{The IRC and the Nucleus of NGC 7793}

The extracted spectra of the IRC are shown in Figures $2(H)$ and $3(K)$, where prominent emission and absorption features are identified. The continuum has been divided out using a low-order function that was fit to each spectrum. A high-order continuum function was not required as high frequency components in the system response were removed during the division by the telluric standard. Spectra of the NGC 7793 nucleus are shown at the bottom of the figures. The NGC 7793 spectra were extracted by summing the signal in a 0.4 arcsec radius aperture, which is where the signal is at least one half of the peak value.

There are obvious differences between the IRC and NGC 7793 spectra. Whereas there is a rich mix of absorption and emission features in the IRC spectra, there is no obvious line emission in the NGC 7793 spectra. This suggests that the nucleus of NGC 7793 does not contain a large population of stars with ages $<10$ Myr. The first and second overtone bands of $\mathrm{CO}$, which are the most prominent absorption features in this wavelength region, are deeper in NGC 7793 than in the IRC. However, a direct comparison of absorption features is affected by nebular continuum emission, which causes absorption features in the IRC spectrum to appear weaker than they would be in the absence of this emission. The contribution made by continuum emission to the spectra can be estimated in a purely empirical 


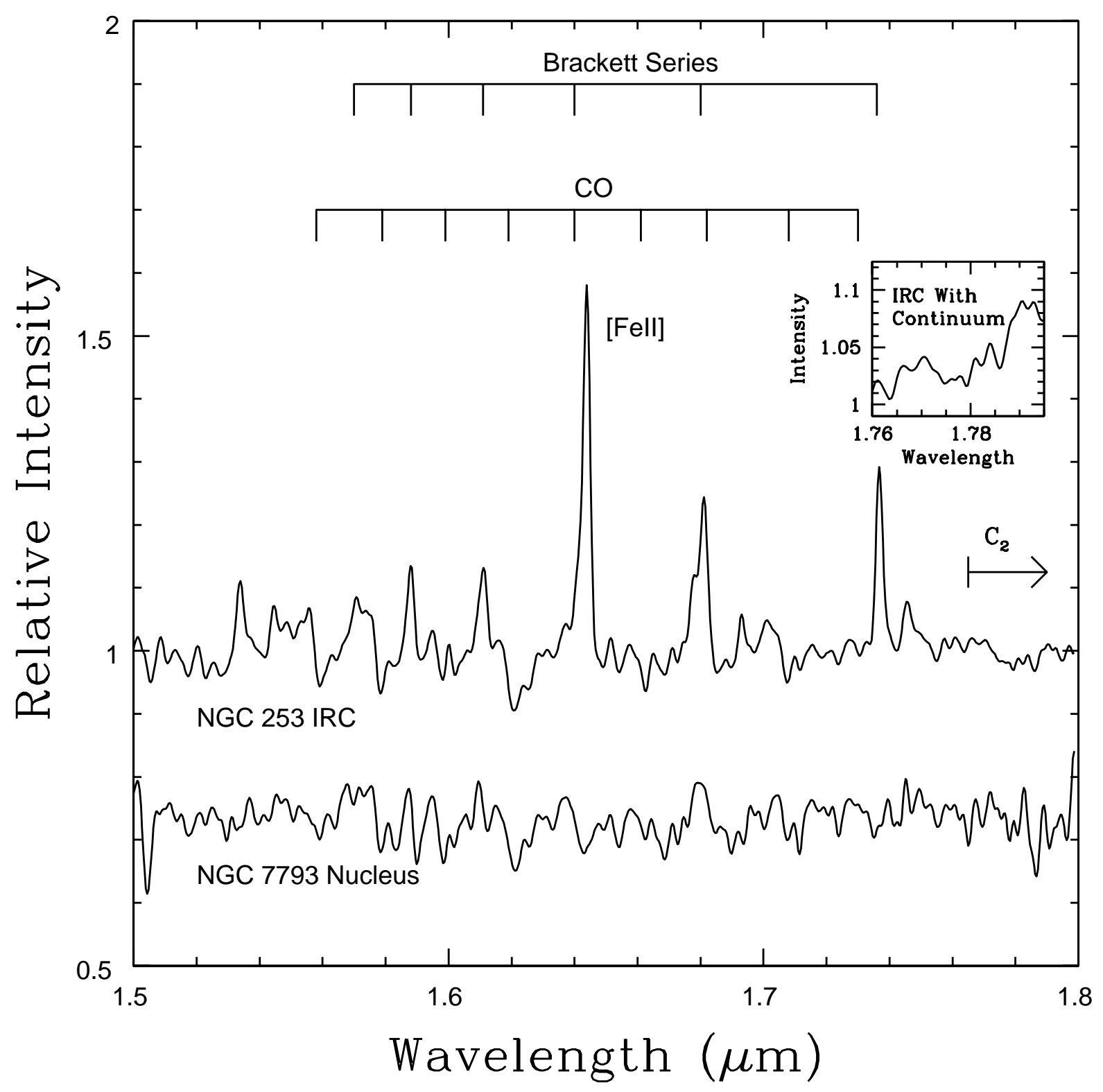

Fig. 2.- $H$-band spectra of the IRC and the nucleus of NGC 7793. The IRC spectrum was obtained by combining signal in a 0.4 arcsec radius circular gather that is centered on the IRC. The spectra have been shifted along the vertical axis for display purposes. The inset shows the IRC spectrum before continuum removal in the wavelength interval where $\mathrm{C}_{2}$ absorption is expected. The absorption trough that is evident in the continuumcorrected spectrum is clearly seen in the inset, indicating that this feature is not an artifact of continuum removal. 


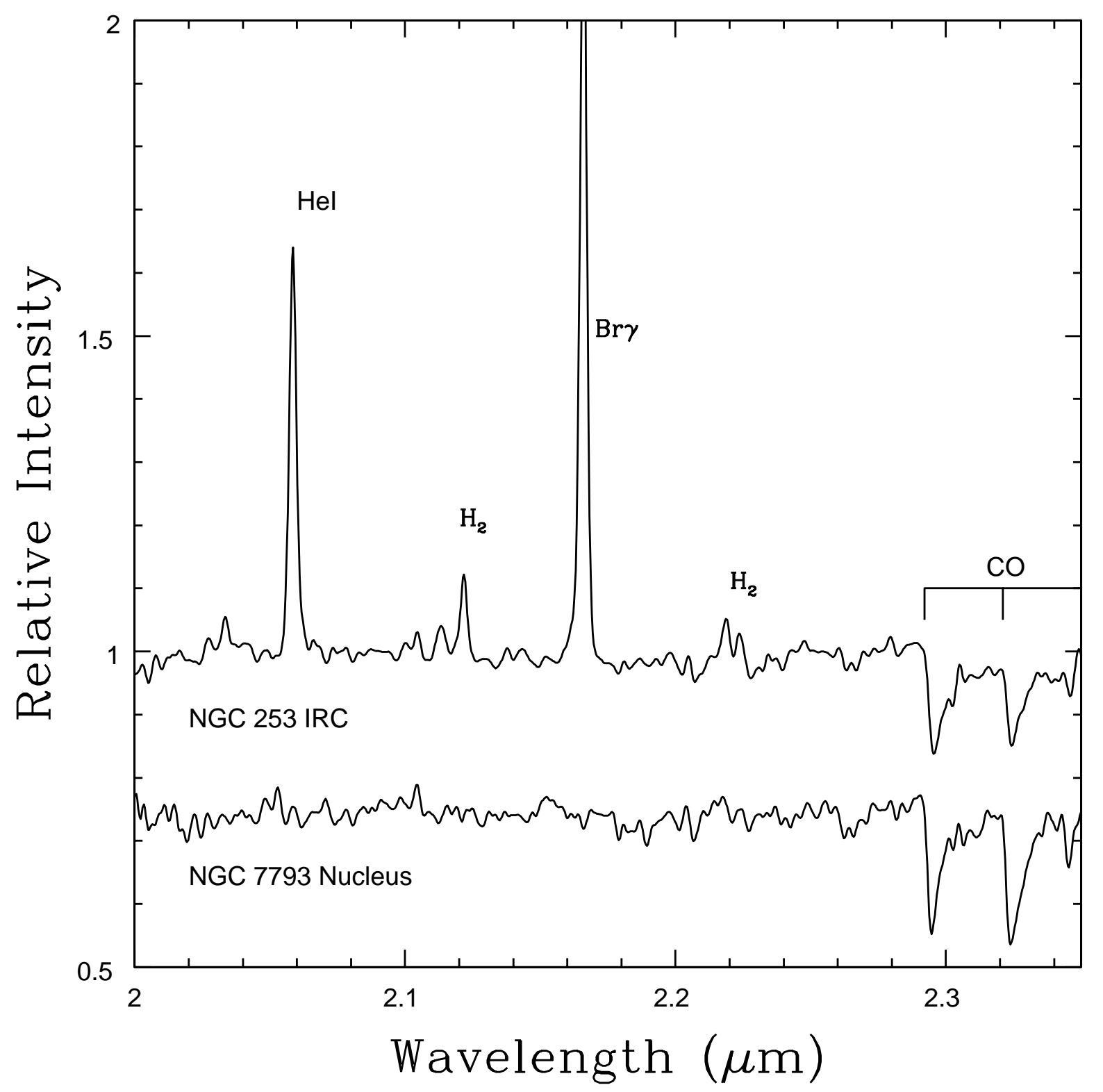

Fig. 3.- Same as Figure 2, but showing $K$-band spectra. Note that the CO bands in the IRC spectrum are not as deep as in the NGC 7793 spectrum. 
manner using the line and continuum measurements made by Armand et al. (1996), who observed five compact Galactic HII regions. The compact morphology of these HII regions makes them areas of concentrated line emission, and so at least some of the conditions near the center of NGC 253 may be replicated, albeit at much smaller levels of activity.

The relative strengths of emission lines and the continuum depends on environmental properties such as the temperatures of the ionizing stars and the density of gas and dust. One way to identify a reference HII region for comparison with the IRC would be to consider the ultraviolet spectral-energy distribution (SED), as this is the wavelength range where the photons that power the emission originate. However, such a comparison is not possible for NGC 253 given the heavy obscuration towards the center of that galaxy. Photometric measurements made in the MIR, where the signal is dominated by thermal emission from dust that is heated by hot stars, offer an alternative. Both the hardness of the radiation field and the properties of the dust affect the MIR emission, and a template HII region was selected based on the W1 - W2 color obtained from WISE (Wright et al. 2010) All Sky Survey images.

The W1-W2 color of the HII region IRAS 12073-6233 matches the W1-W2 color of the center of NGC 253 to within 0.06 magnitudes. In contrast, IRAS 17200-3550 and 17455-2800 have W1-W2 colors that are 0.3 - 0.4 magnitudes redder than the center of NGC 253. Thus, IRAS 12073-6233 was selected to serve as the template for estimating the contribution made by nebular continuum emission to the NIR light from the IRC.

The Br $\gamma$ line width in the extracted IRC spectrum is $0.02 \mu \mathrm{m}$, and so - using the entries for IRAS 12073-6233 in Table 1 and 2 of Armand et al. (1996) - the nebular continuum is $\sim 6 \%$ of the mean strength of the $\operatorname{Br} \gamma$ line flux. The nebular continuum then accounts for $\sim 10 \%$ of the signal from the IRC in $K$. The Starburst99 models summarized in Figure 8 of Reines et al. (2010) predict that this level of continuum emission near $2 \mu \mathrm{m}$ is expected for a system with an age $6-8$ Myr. 
Rather than identifying a single HII region to serve as a reference object, an alternate approach might be to combine the results for all of the HII regions in the Armand et al. (1996) sample to obtain a mean line-to-continuum emission ratio. In fact, the ratio of the $\mathrm{Br} \gamma$ to continuum strength in IRAS 12073-6233 is close to the mean value of all 5 HII regions. Hence, adopting mean values from all HII regions would yield similar results to those obtained using IRAS 12073-6233 alone.

A $10 \%$ contribution from the nebular continuum has a non-trivial effect on the absorption spectrum. This is demonstrated in Figures 4 and 5, where a featureless continuum that accounts for $10 \%$ of the total light has been added to the NGC 7793 spectra. Not surprisingly, the addition of this continuum weakens the absorption features in NGC 7793, producing a better match with the IRC spectra.

The agreement between the absorption features in the IRC and the NGC 7793 spectra with a $10 \%$ contribution from nebular continuum is examined in Figures 4 and 5, where the differences between the IRC and the NGC 7793 nucleus + continuum spectra are shown. Subtracting the NGC7793 + continuum spectrum from the IRC spectrum results in a reasonable suppression of absorption features in the $H$-band. Weak emission features in the NGC 253 spectrum can also be more cleanly identified when the absorption spectrum is suppressed.

The absorption features in the $K$-band are not as well matched as those in the $H$-band. In particular, the residuals near the $2.32 \mu \mathrm{m}(3-1) \mathrm{CO}$ band indicate that this feature is stronger in the NGC 7793 spectrum than in the IRC spectrum, although the residuals near the $2.295 \mu \mathrm{m}(2-0)$ band indicate better agreement. A wavelength-dependent trend in the residuals could result if there is a significant thermal component in the IRC continuum. The experiments considered here assume a nebular component that contributes a fixed percentage of the light. In fact, there is significant thermal emission associated with star-forming knots in the central regions of NGC 253 (e.g. Fernandez-Ontiveros et al. 2009), 


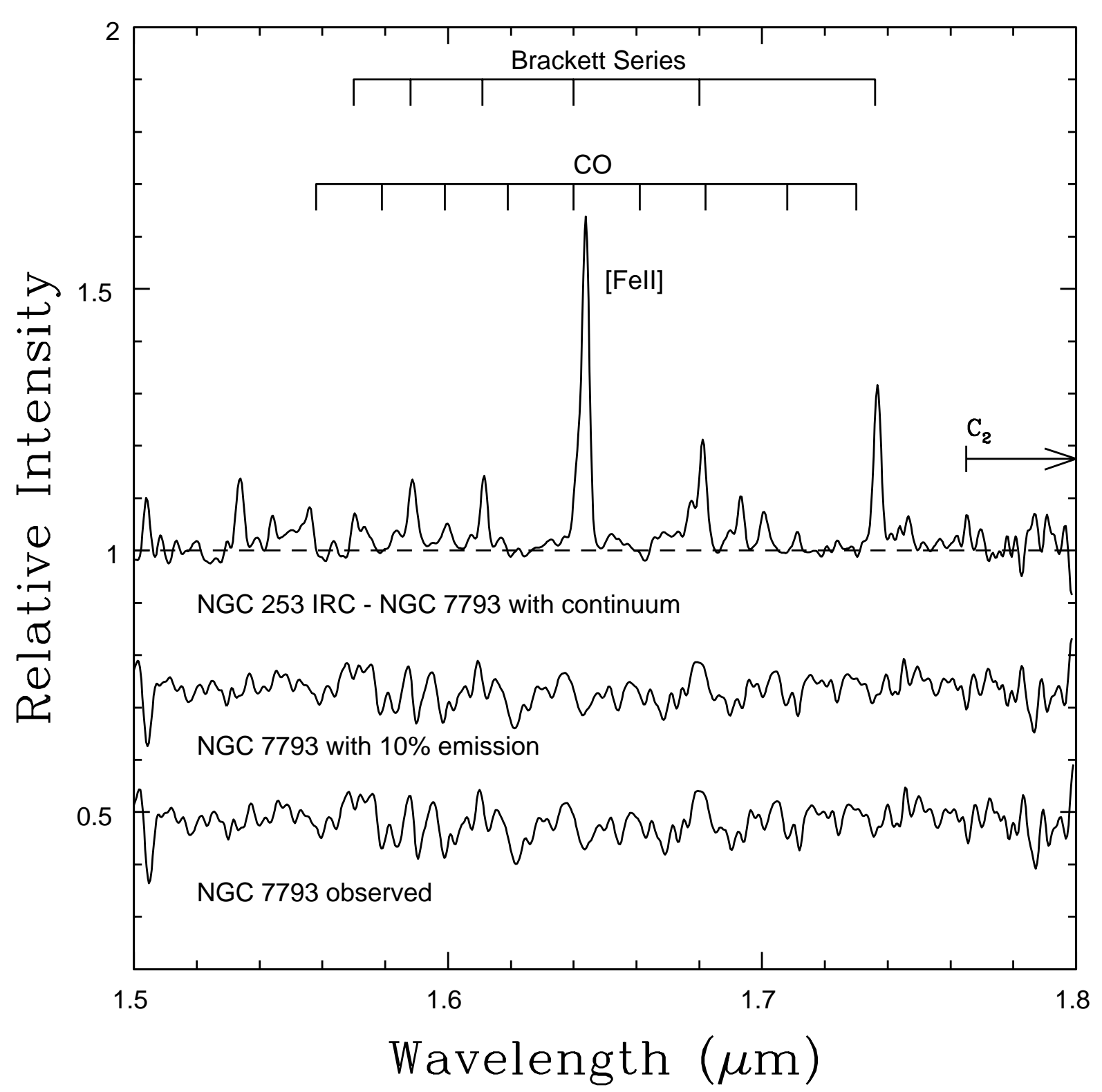

Fig. 4.- Comparing absorption and emission features in the NGC 7793 and NGC 253 IRC $H$-band spectra. The observed spectrum of the NGC 7793 nucleus is shown at the bottom. The middle spectrum shows the result of adding a featureless continuum that accounts for $10 \%$ of the light to the NGC 7793 spectrum. The top spectrum is the difference between the IRC and the NGC 7793 + continuum spectra; the dashed line indicates zero signal. The absorption features in the NGC $7793+$ continuum spectrum appear to match the depths of those in the NGC 253 IRC spectrum. 


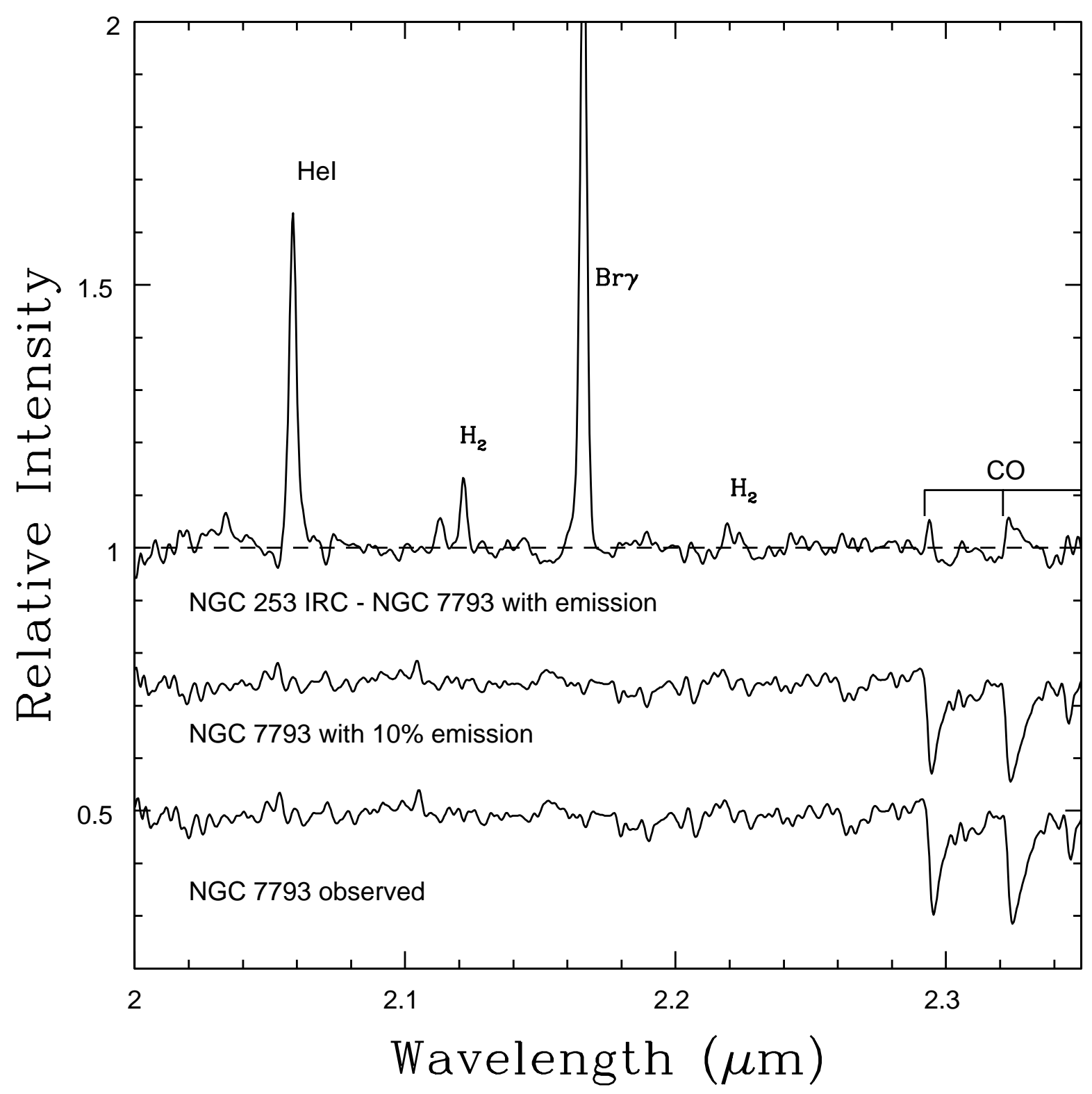

Fig. 5.- Same as Figure 4, but showing $K$-band spectra. The tendency for the residuals to increase towards longer wavelengths when $\lambda>2.3 \mu$ m may be a consequence of emission from hot dust near the center of NGC 253. 
and this emission may contribute significantly near the long wavelength end of the $K$-band. The residuals near $2.32 \mu \mathrm{m}$ in Figure 5 might then be the consequence of failing to account for thermal emission.

The comparisons in Figures 4 and 5 ostensibly suggest that the brightest red stars in the IRC have spectroscopic properties that more-or-less match those in the nucleus of NGC 7793. This agreement is somewhat surprising, as it suggests similarity in the red stellar content. More specifically, if the NIR light in both systems is dominated by red stars then the agreement in line strengths could indicate comparable metallicities. However, the total $K$ magnitudes of these galaxies differ by $\sim 3$ magnitudes, in the sense that NGC 253 is the brighter of the two (Jarrett et al. 2003), and so the IRC might be expected to have a higher metallicity than the nucleus of NGC 7793. Perhaps the gas from which stars in the IRC are forming is moderately metal-poor, possibly having originated in an accreted companion. However, a problem with this speculation is that there is no evidence for metal-poor gas outside of the IRC. Indeed, the $\mathrm{CO}$ bands in the area around the IRC are deeper than those in the nucleus of NGC 7793 (Section 4.2), and have depths that are consistent with a super-solar metallicity (Section 6).

A basic assumption in the preceeding paragraph is that the NIR continuum in the IRC is dominated by red stars. However, if the contribution from hot stars to the total NIR light is sufficiently large then absorption features from red stars will be veiled by the nearly featureless stellar continuum associated with OB stars. This can occur if there are large numbers of stars with ages $<8 \mathrm{Myr}$, as RSGs do not form in this age range - the composite SED of such a young population is then dominated by hot stars. In fact, it is evident from the right hand panel of Figure 1 that the IRC has relatively blue NIR colors. The effect of a contribution from a large population of hot stars to the NIR spectrum of the IRC is investigated further in Section 6.

The Ballick-Ramsey $\mathrm{C}_{2}$ bandhead near $1.78 \mu \mathrm{m}$ is a prominent absorption feature in $\mathrm{C}$ 
star spectra, and so might be expected in the spectrum of a system that experienced largescale star formation during intermediate epochs. In fact, an absorption trough with a depth of a few percent is seen in the IRC spectrum near $1.78 \mu \mathrm{m}$. Absorption at this wavelength is also seen in the East and West spectra (Section 4.2).

The absorption trough near $1.78 \mu \mathrm{m}$ is not an artifact of continuum removal. This is demonstrated in the Figure 2 inset, where the IRC spectrum between 1.76 and $1.79 \mu \mathrm{m}$ without continuum removal is shown. The absorption trough near $1.78 \mu \mathrm{m}$ is clearly seen in the spectrum with the continuum in place. Absorption near $1.78 \mu \mathrm{m}$ is also evident in the $H$-band IRC spectrum in Figure 2 of Kornei \& McCrady (2009).

The spectra of $\mathrm{K}$ and $\mathrm{M}$ giants do not show obvious absorption features at this wavelength (Rayner et al. 2009). However, it is still not obvious that $\mathrm{C}_{2}$ has been detected in the IRC, given the very high levels of extinction towards the center of NGC 253. Engelbrecht et al. (1998) estimate that $\mathrm{A}_{V}=9.2 \pm 0.7$ within 7.5 arcsec of the nucleus based on the Brackett decrement, and $A_{V}=17-19$ from NIR colors. Kornei \& McCrady (2009) estimate that $\mathrm{A}_{V}=18 \pm 3$ from [FeII] lines, and $\mathrm{A}_{V} \sim 12$ from NIR colors. Because of the substantial obscuration, there will likely be significant absorption by diffuse interstellar bands (DIBs). Geballe et al. (2011) discuss the detection of DIBs between 1.77 and $1.79 \mu \mathrm{m}$ in the spectra of Galactic stars with $\mathrm{A}_{V} \sim 20$ magnitudes, and an absorption feature at $1.793 \mu \mathrm{m}$ is the deepest DIB discovered in their spectrum of GCS3-2.

Given that the extinction towards the center of NGC 253 is comparable to that towards the stars examined by Geballe et al. (2011) then the $\mathrm{C}_{2}$ wavelength region likely also contains DIB signatures. However, these signatures are not expected to be present in the nuclear spectrum of NGC 7793, where the line-of-sight extinction is much lower than towards the IRC. The residuals in Figure 4 suggest that the IRC and the NGC 7793 nucleus spectra have similar levels of absorption in the wavelength interval that hosts the $\mathrm{C}_{2}$ band. 


\subsection{The Circumcluster Spectrum}

The spectra of NGC 253 East and West are shown in Figures 6 ( $H$-band) and 7 ( $K$-band). The difference between the two spectra is shown at the bottom of each figure. The largest differences between the spectra occur at wavelengths where there are strong emission lines, with many (but not all) of these lines being stronger in NGC 253 East, which is an area where bluer F128N-F164N colors are seen (Figure 1). As for absorption features, the CO bands in the East and West spectra have similar depths. The scatter in the $1.76-1.78 \mu \mathrm{m}$ interval, which covers part of the wavelength range where $\mathrm{C}_{2}$ absorption might be expected, is similar to that seen at other wavelengths. However, there is much larger scatter in the differenced spectra at wavelengths $>1.78 \mu \mathrm{m}$. This likely is due to the amplification of the noise that occurs near $1.8 \mu \mathrm{m}$ when correcting for telluric water absorption.

Many absorption and emission features in the $H$ and $K$ spectra of NGC 253 East and West have markedly different strengths when compared with their counterparts in the IRC spectra. Emission lines of H, HeI, and [FeII] in NGC 253 East and West are weaker than in the IRC. This suggests that while very young stars are present outside of the IRC, their density when measured with respect to the stars that dominate the NIR continuum is lower than in the IRC. $\mathrm{H}_{2}$ emission is an exception. The strongest $\mathrm{H}_{2}$ features in NGC 253 East and West are comparable to or even stronger than those in the IRC, with no difference between NGC 253 East and West. This suggests that the source of the $\mathrm{H}_{2}$ excitation across the IFU field is well-mixed with the stars that dominate the NIR light when averaged over arcsec angular scales. As noted by Rosenberg et al. (2013), this is consistent with the stars that power $\mathrm{H}_{2}$ emission having lower masses than those that power $\mathrm{Br} \gamma$ and $\mathrm{HeI}$ emission, as these objects would then have had more time to mix throughout the central regions of NGC 253. 


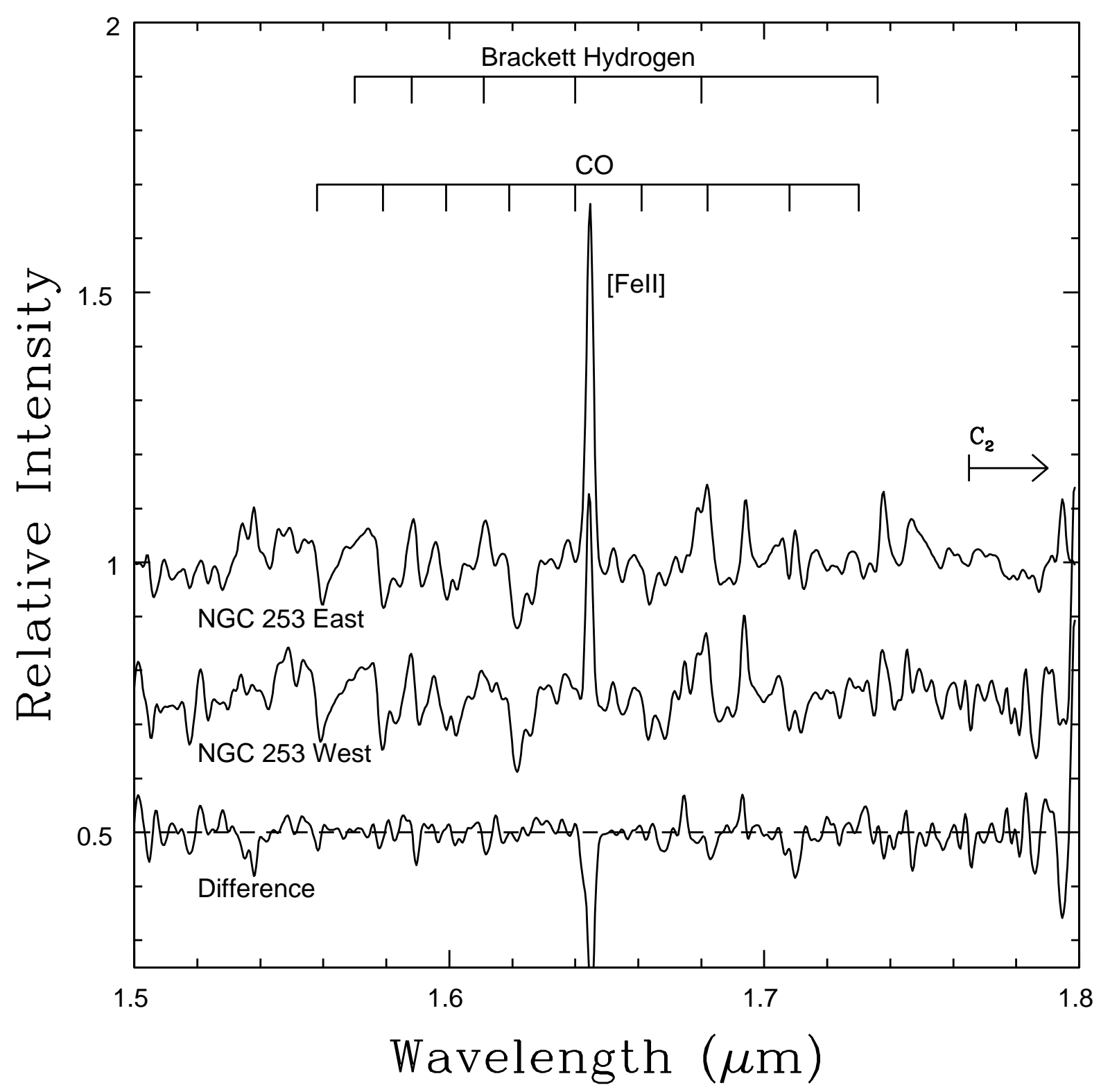

Fig. 6. - Spectra of NGC253 East and West. The continuum has been divided out of each spectrum, and the difference between the two spectra is shown at the bottom of the figure. By far the largest differences are associated with [FeII], with this line being stronger in NGC 253 East. In contrast, the second-overtone CO bands in the East and West spectra have similar depths, and these are in turn deeper than in the IRC. 


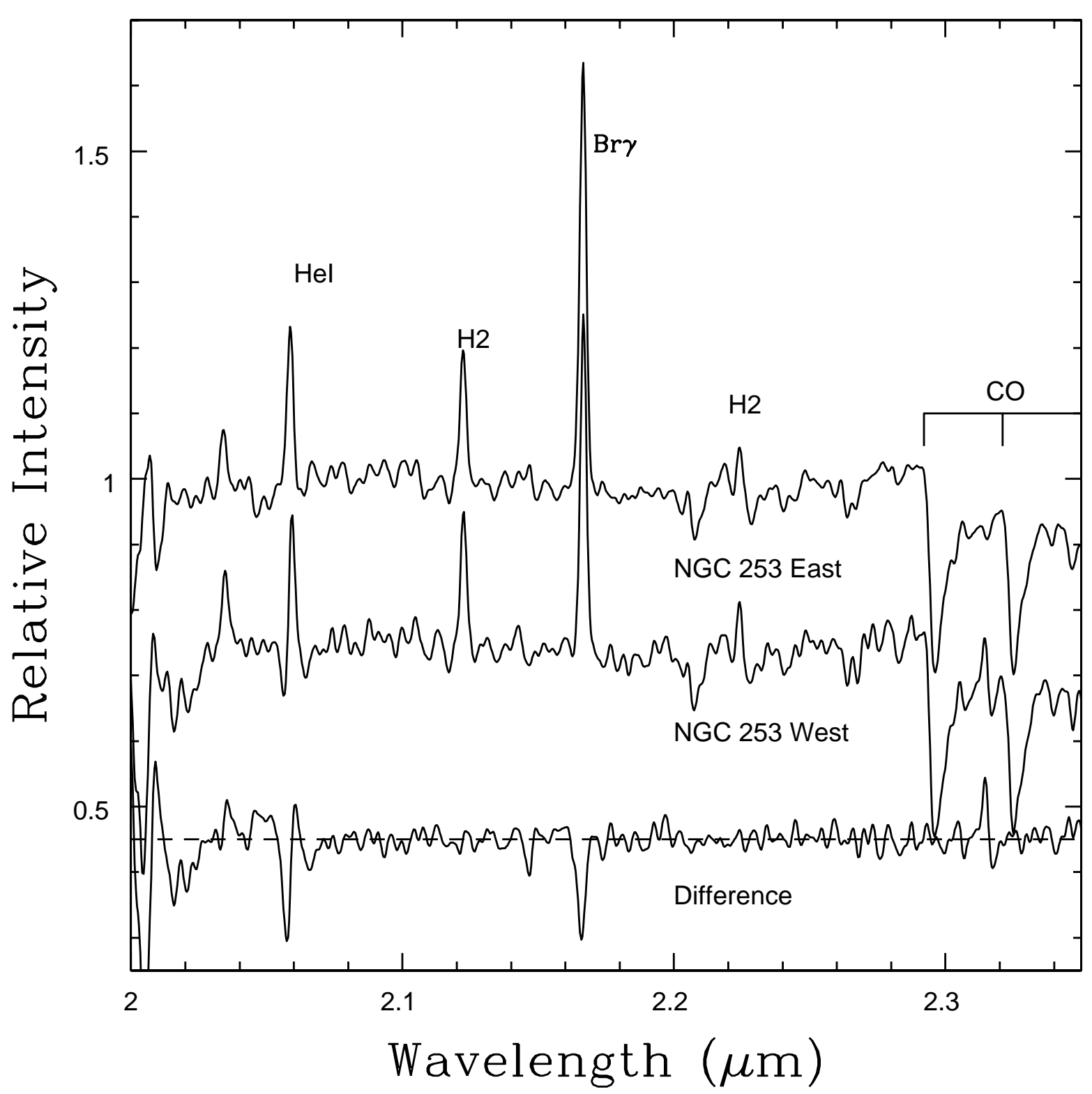

Fig. 7.- Same as Figure 6, but showing $K$-band spectra. The East and West spectra have significantly different $\mathrm{Br} \gamma$ and HeI strengths, with both lines being stronger in NGC 253 East. This is in contrast to the $\mathrm{H}_{2}$ lines, which have remarkably similar strengths in NGC 253 East and West. The $\operatorname{Br} \gamma$ and HeI features in both spectra are weaker than in the IRC. The first overtone CO bands in NGC 253 East and West have similar depths, and these are in turn much deeper than in the IRC. 
The CO bands in NGC 253 East and West are deeper than in the IRC, and this is most noticeable for the first-overtone transitions. It is unlikely that this difference is a consequence of veiling of the IRC spectrum by non-stellar continua, which was discussed in Section 4.1. In order for nebular continuum emission alone to veil the CO bands in NGC 253 East and West so that they match what is seen in the IRC then the contribution from this emission would

have to be $\sim 7 \times$ higher than estimated for the IRC in Section 4.1. Thermal emission also can not explain the differences in the depths of the $2.2 \mu \mathrm{m}$ features, as the second overtone bands in the $H$-band circumnuclear spectra are deeper than in the IRC. The impact of a thermal contribution at these shorter wavelengths is negligible. Rather than a non-stellar continuum, the differences between the IRC and East and West spectra could be due to large numbers of hot stars in the IRC, and this is considered in Section 6.

\section{THE SPATIAL DISTRIBUTION OF SPECTRAL FEATURES}

The spectra that were investigated in Section 4 were constructed by combining data over large portions of the area sampled by the IFU. This binning was done at the expense of angular resolution to boost the $\mathrm{S} / \mathrm{N}$ ratio. However, there are some features (most of them emission lines) that are sufficiently strong that their projected distribution can be examined without binning. The on-sky behaviour of these features is examined in this section.

\subsection{Continuum and Absorption Features}

There are wavelength intervals that are free of strong emission features, and the signal in these can be used to map the distribution of light from the stars that dominate the NIR continuum free of contamination from prominent emission lines. Two such wavelength intervals are between $1.72 \mu \mathrm{m}$ and $1.73 \mu \mathrm{m}$ (hereafter referred to as Hcont) and $2.24 \mu \mathrm{m}$ and $2.28 \mu \mathrm{m}$ (hereafter Kcont). The distribution of light in Kcont and the Hcont-Kcont color are 
shown in Figure 8. The Kcont and Hcont-Kcont images show features that match those in Figure 1. The Hcont-Kcont color of the IRC is $0.3-0.4$ magnitudes bluer than that of the surroundings.

Mapping the distribution of absorption features provides important information about stellar content and the mix of stellar types. However, obtaining maps for all but the deepest absorption features is problematic given the modest $\mathrm{S} / \mathrm{N}$ ratio in spaxals that are external to the IRC. The first overtone CO bands, which are the deepest absorption features in these spectra, are an exception. The depth of the $(2,0)$ CO band was measured in the wavelength interval $2.29 \mu \mathrm{m}-2.31 \mu \mathrm{m}$, and a CO index was constructed by dividing the mean signal in this wavelength interval by the Kcont flux. The spatial behaviour of the CO index near the center of NGC 253 is shown in the lower panel of Figure 8.

The spectroscopic properties of the IRC differ from those in the surrounding area. The $\mathrm{CO}$ index in the IRC is $10-15 \%$ weaker than in the surroundings, in agreement with the extracted spectra discussed in Sections 4.1 and 4.2. It is perhaps surprising that there are only minor variations in the CO index in the area external to the IRC given the large range of F128N-F164N colors that are seen there in Figure 1. An exception is the pocket of deep CO absorption near the northern edge of the IFU, which has a slightly redder Hcont-Kcont color than its surroundings. If there are large numbers of RSGs in this area that have ages $\sim 10 \mathrm{Myr}$, and so are younger than those that populate most of the IFU field (Section 6), then this area might be expected to have deeper CO bands and redder colors.

Kcont, Hcont-Kcont, and CO images were also constructed for the central regions of NGC 7793. Apart from an obvious nucleus in the Kcont image, these images do not reveal significant structure, and so are not shown here. In particular, the CO index of the NGC 7793 nucleus is comparable to that in the circumnuclear area, which is contrary to what is seen in NGC 253. 


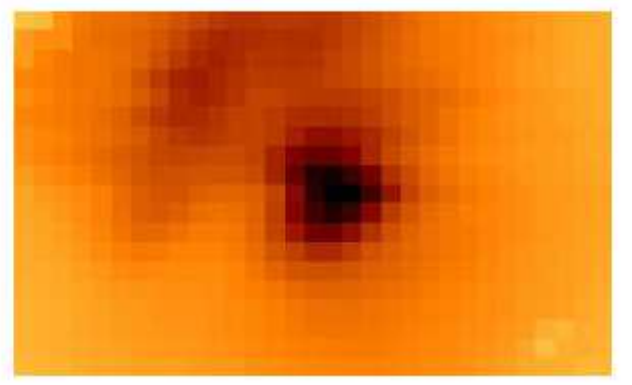

\section{Kcont}

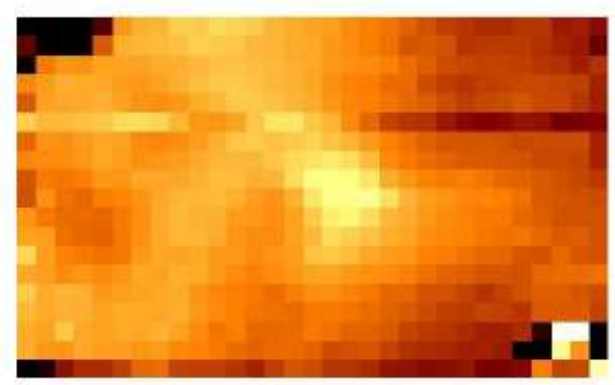

H-K

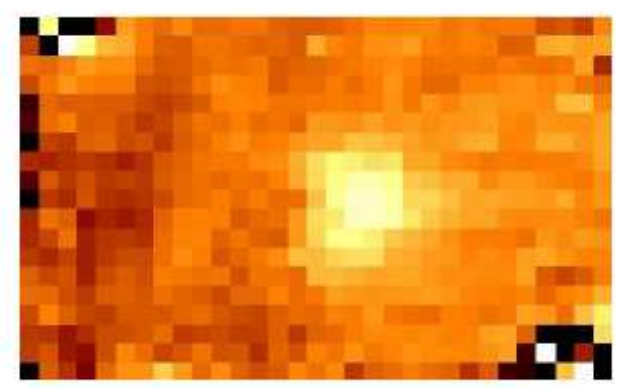

CO

Fig. 8. - Comparing the distribution of stellar continuum light and the depth of the $(2,0) \mathrm{CO}$ band. Each image covers $4.9 \times 3.2$ arcsec, with North to the left and East at the top. Darker shading in the Hcont-Kcont image indicates redder colors. Hcont-Kcont varies throughout the area surveyed, likely due to non-uniform extinction and variations in stellar content. The IRC is $\sim 0.3-0.4$ magnitudes bluer than the surroundings. The $\mathrm{CO}$ index, which gauges the strength of the $(2,0) \mathrm{CO}$ band head near $2.3 \mu \mathrm{m}$, is shown in the bottom panel. Smaller $\mathrm{CO}$ indices (i.e. shallower $\mathrm{CO}$ bands) are displayed with lighter colors. The $\mathrm{CO}$ index is weakest in the IRC, and is strongest near the northern boundary of the IFU. The CO index of the IRC is $10-15 \%$ lower than in the surroundings. 


\subsection{Line emission}

The emission lines in the NGC 253 spectra probe a diverse range of environments, and so may not track light from the stars that dominate the NIR continuum. In this section various emission line indices normalized to Kcont are used to examine differences in the distribution of the line-emitting sources and the underlying stellar continuum. Comparisons of this nature provide insights into the mix of stellar types throughout the area sampled with the IFU, including the identification of areas that have very young luminosity-weighted ages.

Spectral indices were computed for four emission lines using the wavelength intervals listed in Table 1. The line measurements were made in the wavelength interval between the points where the wings of the line merges with the adjacent continuum in these data. The continuum is measured on both sides of each line, and the mean continuum contribution was subtracted to obtain an index that tracks only line emission.

\subsection{1. $B r \gamma / K$ cont}

The ratio of the $\operatorname{Br} \gamma$ and Kcont indices is shown in the upper left hand corner of Figure 9. If $\operatorname{Br} \gamma$ and Kcont have the same projected distributions then the image would be featureless. However, the $\operatorname{Br} \gamma$ to Kcont ratio peaks in the outer areas of the IRC, with the

\begin{tabular}{lccc}
\hline \hline Feature & $\begin{array}{c}\text { Line } \\
(\mu \mathrm{m})\end{array}$ & $\begin{array}{c}\text { Continuum 1 } \\
(\mu \mathrm{m})\end{array}$ & $\begin{array}{c}\text { Continuum 2 } \\
(\mu \mathrm{m})\end{array}$ \\
\hline $\mathrm{Br} 13$ & $1.6136-1.6449$ & $1.6088-1.6131$ & $1.6454-1.6499$ \\
{$[\mathrm{FeII}]$} & $1.6413-1.6485$ & $1.6374-1.6408$ & $1.6490-1.6524$ \\
$\mathrm{HeI}$ & $2.0553-2.0637$ & $2.0508-2.0547$ & $2.0643-2.0675$ \\
$\mathrm{Br} \gamma$ & $2.1612-2.1705$ & $2.1429-2.1606$ & $2.1711-2.1796$ \\
\hline
\end{tabular}

Table 1: Wavelengths for Emission Line Measurements 
highest ratios defining an arc that girds the western half of the IRC. Br $\gamma$ emission thus has a more extended spatial distribution than Kcont in and around the IRC.

The $\operatorname{Br} \gamma$ to Kcont ratio also peaks in the north east corner of the IFU field. The $\mathrm{HeI} /$ Kcont and $[\mathrm{FeII}] /$ Kcont ratios are also high in this area (see below). There are pockets with blue F128N-F164N colors in this area (Figure 1), and these may be areas of star-forming activity powering the $\operatorname{Br} \gamma$ emission.

The photons that excite $\mathrm{Br} \gamma$ emission likely originate from $\mathrm{O}$ stars, and Kornei \& McCrady (2009) find that the IRC contains a large number of stars with ages $\sim 6$ Myr based on the strength of $\operatorname{Br} \gamma$ emission. To the extent that $\operatorname{Br} \gamma$ emission tracks young stars then the arc to the west of the IRC is where the ratio of hot young stars to the stars that dominate the NIR stellar continuum is locally highest. It is thus worth noting that the CO index in the western arc of $\operatorname{Br} \gamma$ emission is not weaker than in the main body of the IRC the continuum from hot stellar photospheres evidently does not veil the CO features in the outer regions of the IRC. However, veiling is likely an issue in the main body of the IRC (Section 6).

\subsection{2. $B r 13 / B r \gamma$}

The relative strengths of lines in the Brackett series can be used to investigate reddening, although there are caveats. While the Brackett lines sample wavelengths where the

effects of extinction are greatly diminished when compared with visible wavelengths, there may be areas of extremely high extinction that are largely opaque in the NIR, and measuring the total line-of-sight extinction towards these areas will be problematic. Moreover, while wide wavelength coverage provides the greatest leverage for estimating extinction, the lines progressively weaken towards higher-order transitions, resulting in a trade-off between sensitivity to reddening variations and noise in line strength measurements. In addition, lines 


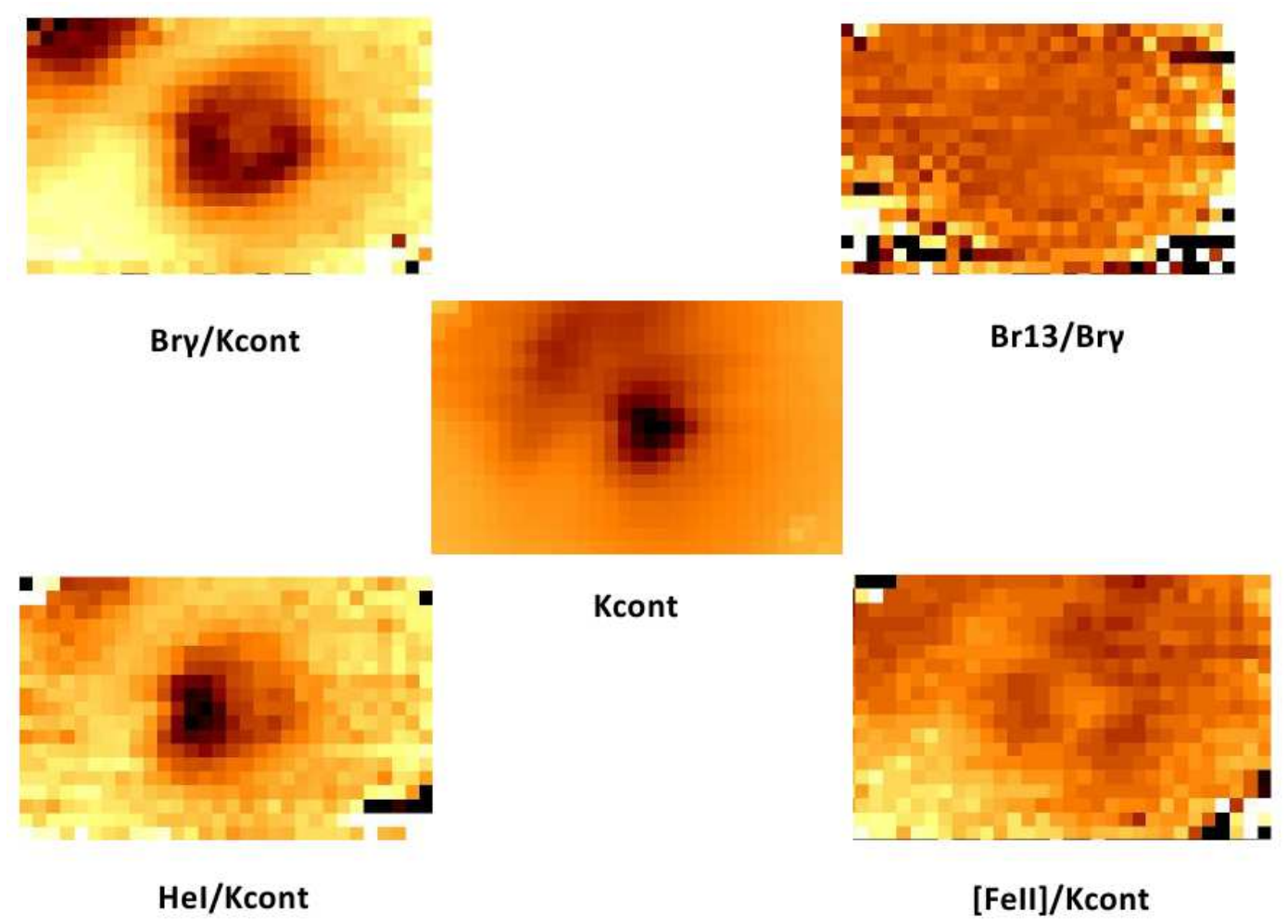

Fig. 9.- Emission line indices. Each panel samples a $4.9 \times 3.2$ arcsec area, with north to the left and east at the top. The Kcont image from Figure 8 is shown in the middle panel. Top left hand corner: The ratio of $\operatorname{Br} \gamma$ to Kcont. $\operatorname{Br} \gamma$ emission has a more extended distribution on the sky than the stars that dominate the $K$ continuum, with the $\operatorname{Br} \gamma /$ Kcont ratio peaking along the western edge of the IRC, as well as in the north east corner of the IFU field. Top right hand corner: The ratio of $\mathrm{Br} 13$ to $\mathrm{Br} \gamma$. There is a tendency for this ratio to decrease with increasing distance from the center of the IRC. Lower left hand corner: The ratio of HeI to Kcont. HeI emission has a more extended distribution than the light from the stars that dominate the NIR continuum near the IRC. The HeI/Kcont ratio peaks near the northern edge of the IRC, suggesting that this is an area where Wolf-Rayet stars are concentrated with respect to the stars that dominate Kcont. The area where the HeI/Kcont ratio is highest coincides with a peak in the $\mathrm{Br} \gamma / \mathrm{Kcont}$ ratio. Lower right hand corner: The ratio of $[\mathrm{FeII}]+\mathrm{Br} 12$ to Kcont. Areas of residual [FeII] emission bracket the IRC. The excess [FeII] emission to the south east of the IRC is located along a ridge in the $\mathrm{Br} \gamma /$ Kcont ratio, although the $\operatorname{Br} \gamma /$ Kcont ratio there is lower than in the $\operatorname{Br} \gamma$ arc to the west of the IRC. 
that are at different wavelengths may not originate at a common optical depth, skewing extinction estimates. Finally, relative line strengths depend on the electron temperature and density, and if these vary substantially across a region then the Brackett decrement will not be a faithful probe of extinction.

The ratio of the $\operatorname{Br} 13$ and $\mathrm{Br} \gamma$ indices is shown in the upper right hand corner of Figure 9. Br13 was selected for comparison with $\operatorname{Br} \gamma$ by balancing the desire for a wide wavelength baseline against line strength. There are also well-defined continuum intervals on either side of Br13.

While the $\operatorname{Br} 13$ to $\operatorname{Br} \gamma$ ratio shows only subtle variations and there is considerable noise in this ratio outside of the IRC, aperture measurements reveal a systematic tendency for the $\operatorname{Br} 13$ to $\operatorname{Br} \gamma$ ratio to decrease with increasing distance from the IRC. If the electron temperature and density are more-or-less constant across the area sampled then this behaviour suggests that extinction becomes lower with increasing distance from the IRC. This runs counter to the trend for color to become redder outside of the IRC (e.g. Figure 1). We speculate that the Brackett decrement near the IRC is tracking differences in ionization properties, rather than differences in line-of-sight extinction. In fact, the ratio of HeI line emission strength to Kcont - discussed in the next section - changes with distance from the center of IRC, indicating that very hot stars are not uniformly distributed near the IRC.

\subsubsection{HeI/Kcont}

Prominent HeI 2.06 $\mu \mathrm{m}$ emission suggests that W-R stars are present in and around the IRC. The presence of these stars indicates that there has been large-scale star formation near the center of NGC 253 within the past few Myr. The ratio of the HeI and Kcont indices is

shown in the lower left hand corner of Figure 9. The W-R stars that presumably power the HeI emission have a distribution that does not follow the stars that dominate Kcont. There 
is an area of localized high HeI emission with respect to Kcont to the north west of the IRC, and this is likely where the ratio of $\mathrm{W}-\mathrm{R}$ stars to the stars that dominate the continuum near the IRC is highest. In addition to being an area of excess (when compared with stellar light) $\operatorname{Br} \gamma$ and $[\mathrm{FeII}]$ emission (see below), the pocket of enhanced HeI emission is close to an area with blue F128N-F164N color in Figure 1, which is consistent with there being a concentration of hot, blue stars.

\subsection{4. $[\mathrm{FeII}] /$ Kcont}

[FeII] emission is excited in shocks, and is usually associated with SNRs in star-forming environments. While the $1.64 \mu \mathrm{m}$ [FeII] line is one of the strongest emission features in the NGC 253 NIR spectrum, it is blended with Br12 in the GNIRS data. Based on the peak intensity of the $[\mathrm{FeII}]+\mathrm{Br} 12$ blend, coupled with the relative strengths of $\mathrm{Br} 11$ and $\mathrm{Br} 13$, it appears that Br12 contributes $\sim 20-25 \%$ of the total signal in the [FeII] index as defined in Table 1. This level of contamination will bias the [FeII] index to follow the distribution of Brackett hydrogen emission.

The $[\mathrm{FeII}] /$ Kcont ratio is mapped in the lower right hand corner of Figure 9. As with the other emission indices, $[\mathrm{FeII}]$ does not follow the Kcont light. There are four areas where there are localized peaks in the $[\mathrm{FeII} /] /$ Kcont ratio. One is in the north east corner of the IFU field, where $\mathrm{Br} \gamma / \mathrm{Kcont}$ and $\mathrm{HeI} /$ Kcont also peak. The other three areas surround the IRC. Two of these are along the western edge of the IRC and are close to areas that have a high $\mathrm{Br} \gamma /$ Kcont ratio, and one of these in turn coincides with the peak in the HeI/Kcont ratio. The fourth peak in the $[\mathrm{FeII}] /$ Kcont ratio is located to the south east of the IRC. While the $[\mathrm{FeII}] /$ Kcont ratios in the four regions are comparable, this is not the case for the $\mathrm{Br} \gamma /$ Kcont ratio, indicating that the $[\mathrm{FeII}]$ index does not parrot $\mathrm{Br} \gamma$ emission.

The distributions of $\mathrm{Br} \gamma$, HeI, and [FeII] emission are compared in Figure 10, where 
contours obtained from the $\operatorname{Br} \gamma /$ Kcont and HeI/Kcont images are superimposed on the $[\mathrm{FeII}] /$ Kcont image. The local peaks in the $[\mathrm{FeII}] /$ Kcont ratio around the IRC are offset slightly from those in $\mathrm{Br} \gamma / \mathrm{Kcont}$ and $\mathrm{HeI} / \mathrm{Kcont}$, in the sense that the [FeII] emission peaks at slightly larger distances from the center of the IRC when compared with $\mathrm{Br} \gamma$ or HeI. Such an offset could result if the [FeII] emission is tracking a shock front - presumably powered by $\mathrm{SNe}$ activity - that is propogating outwards from the star-forming regions. We note that Gunthardt et al. (2015) conclude that there is an outflow from the IRC based on the distribution of [NeII] $12.8 \mu \mathrm{m}$ and Si $8.8 \mu \mathrm{m}$ emission.

\section{COMPARISONS WITH MODELS}

Comparisons with model spectra can provide insights into the stellar content in and around the IRC. Highly evolved red stars are the dominant contributors to the photospheric NIR light from simple stellar populations (SSPs) that have ages $\geq 8-10 \mathrm{Myr}$. Uncertainties in the physics associated with the most advanced stages of stellar evolution (e.g. review by Herwig 2005) thus play a significant role in interpreting the spectra of such systems. The well-known age-metallicity degeneracy adds additional uncertainties, as the color (and hence spectroscopic properties) of evolved stars depend on both age and metallicity.

Spectra constructed from SSP models described by Leitherer et al. (1999 - hereafter STARBURST99) and Cordier et al. (2007 - hereafter BaSTI) are compared with the observed spectra of NGC 253 and NGC 7793 in this section. The model spectra have a resolution $\lambda / \Delta \lambda \sim 200$, and so even prominent features such as the first and second-overtone $\mathrm{CO}$ bands are blended with other molecular and/or atomic transitions. The baseline models assume solar metallicities and constant SFRs (cSFRs) from the present day to a time in the past. Given that the NGC 253 and NGC 7793 spectra were not flux calibrated, the comparisons focus on matching the absorption characteristics in continuum-corrected spectra, and no effort is made to estimate a SFR from the models. In any event, an estimate of 


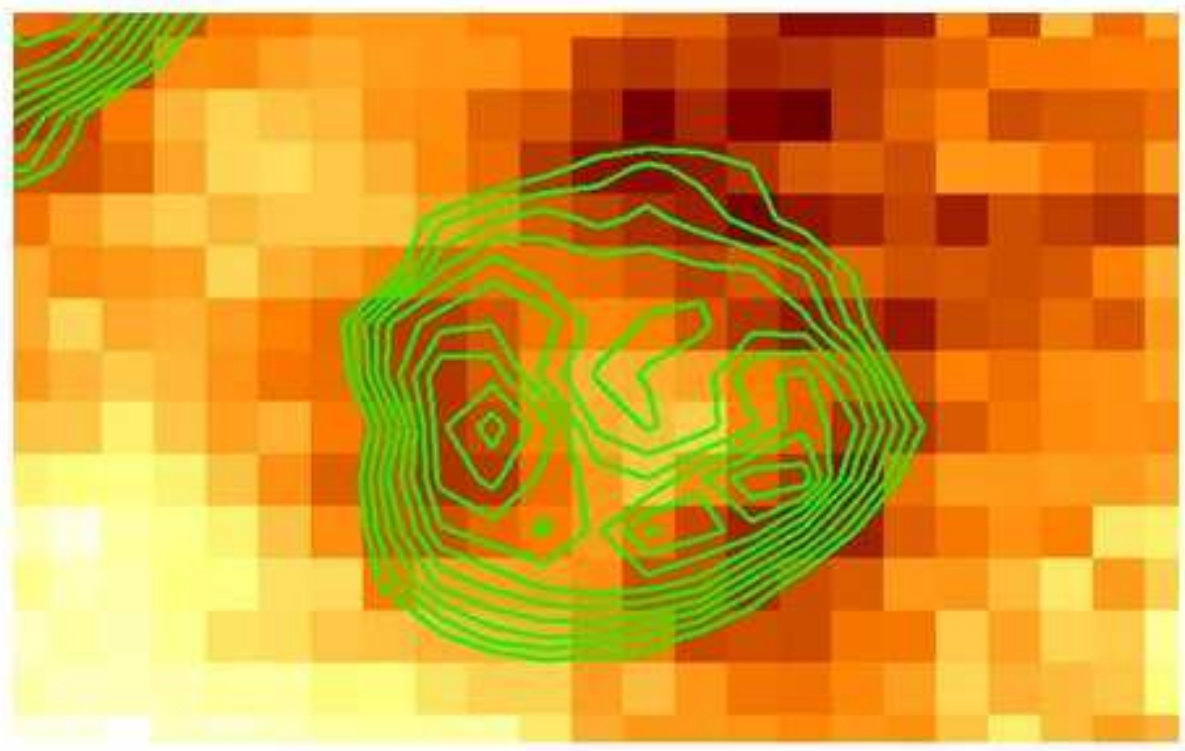

\section{Bry contours}

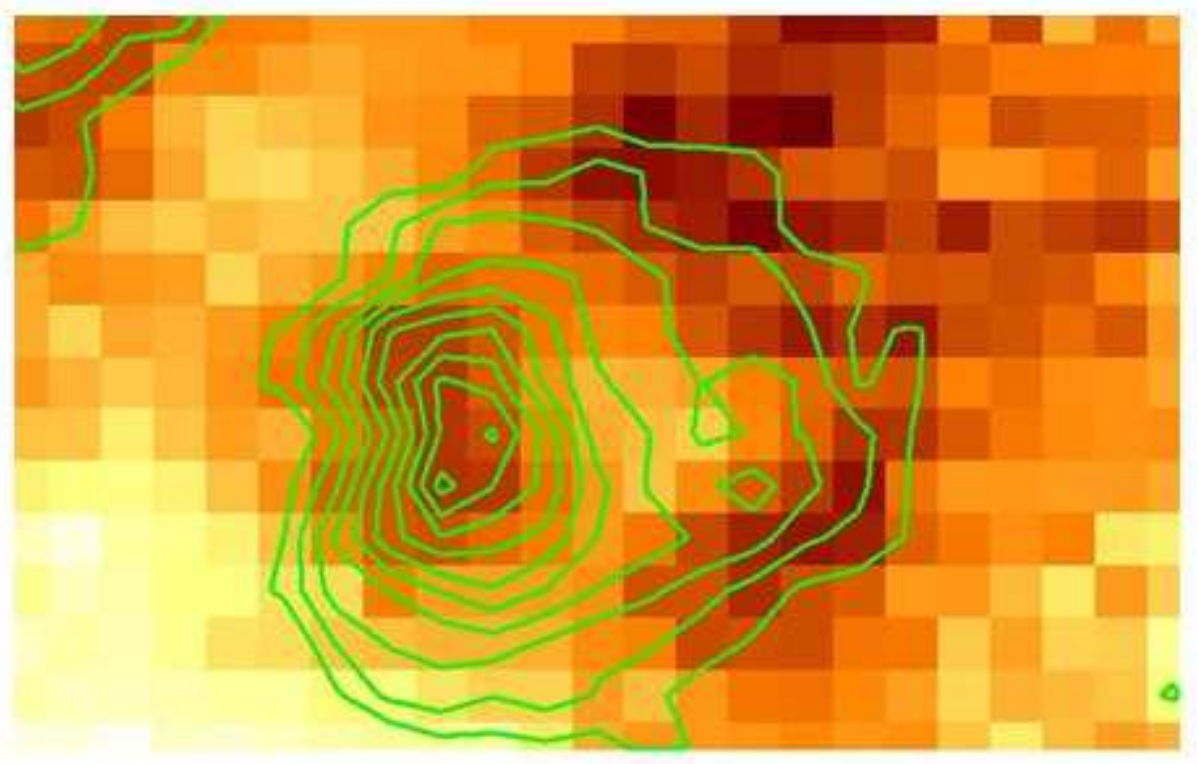

\section{Hel contours}

Fig. 10.- Comparing the distributions of [FeII], $\mathrm{Br} \gamma$, and HeI emission. The base image in each panel is a $4.1 \times 2.3$ arcsec sub-section of the the $[\mathrm{FeII}] /$ Kcont image from Figure 9 . The green contours were obtained from the $\mathrm{Br} \gamma /$ Kcont (top panel) and HeI/Kcont (bottom panel) images in Figure 9. Localized peaks in the [FeII] distribution near the IRC are offset to larger distances from the center of the IRC than the peaks in the $\operatorname{Br} \gamma$ and HeI distributions. 
the SFR depends on the star-forming history ( $\mathrm{SFH}$ ), which is not well-constrained by the NIR spectrum. The models are also used to examine the effect of veiling from very young populations, as this can have a significant impact on the depth of absorption features.

\subsection{The Models}

cSFR models constructed from STARBURST99 SSP spectra are shown in the left hand panels of Figures 11 ( $H$-band) and $12(K$-band). The models have had the continuum removed, and have been shifted vertically for display purposes. The models are based on the solar metallicity Padova isochrones described by Bertelli et al. (1994), with TP-AGB evolution implemented using the procedures described by Vazquez \& Leitherer (2005). A two-component Kroupa (2001) mass function is adopted.

cSFR models constructed from SSP spectra in the BaSTI compilation are shown in the right hand panels of Figures 11 and 12. The model spectra are based on the solar metallicity isochrones described by Pietrinferni et al. (2004), and include TP-AGB evolution as described by Cordier et al. (2007). A Chabrier (2001) mass function is adopted. The BaSTI models do not include systems with ages $<30 \mathrm{Myr}$, and so the STARBURST99 SSP models in that age range were used when constructing the BaSTI cSFR models.

The first and second-overtone $\mathrm{CO}$ bands are the dominant features in the models. These are deepest in the 50 and 100 Myr spectra, reflecting the large contribution to NIR light made by luminous low surface-gravity RSGs at these ages. The differences in the CO band strengths due to age are not large, and the first-overtone CO bands are only a few percent deeper in the 50 and 100 Myr models when compared with models in which star formation continues over a longer time frame. The depths of the CO bands do not change significantly in the cSFR models with ages $\geq 1 \mathrm{Gyr}$, and so the $\mathrm{CO}$ bands contain little information about the SFH of older composite systems. That these features are not sensitive to the SFH 


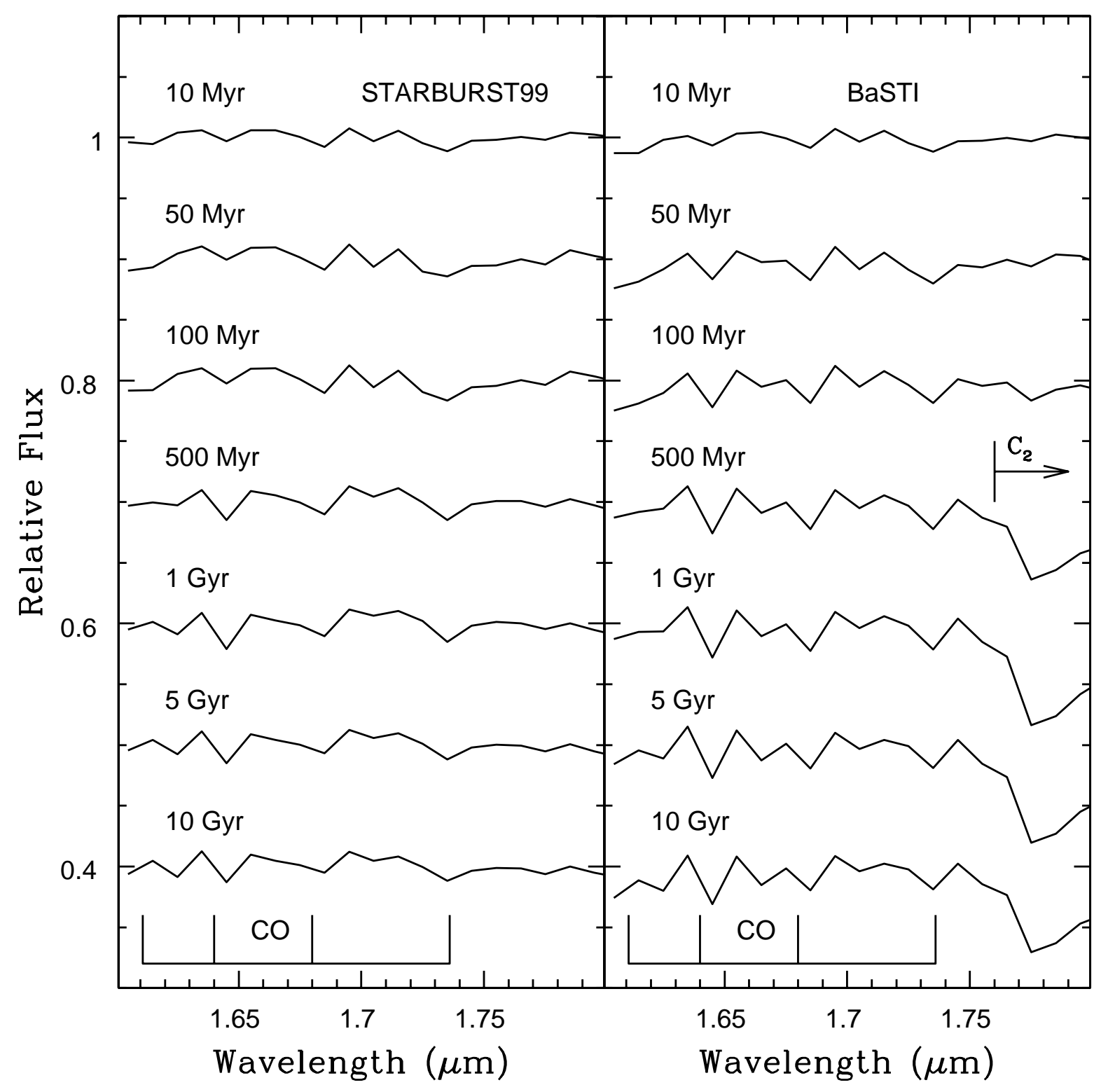

Fig. 11. - Solar metallicity cSFR models constructed from STARBURST99 and BaSTI SSP $H$-band spectra. The models have had the continuum removed, and have a resolution $\lambda / \Delta \lambda \sim 170$. The locations of CO band heads are indicated. The models assume a continuous SFR (cSFR) from the present day to the age indicated; for example, the 1 Gyr model reproduces the integrated spectrum of a system that evolves with a cSFR from 1 Gyr ago up to the present day. The 10 Myr STARBURST99 model is repeated in the right hand panel, as the BaSTI models do not track evolution younger than $30 \mathrm{Myr}$. Changes in age cause variations in the strengths of most features at the few percent level. $\mathrm{C}_{2}$ absorption with a depth of a few percent is seen at wavelengths $>1.77 \mu \mathrm{m}$ in BaSTI models with ages $\geq 500$ Myr. 


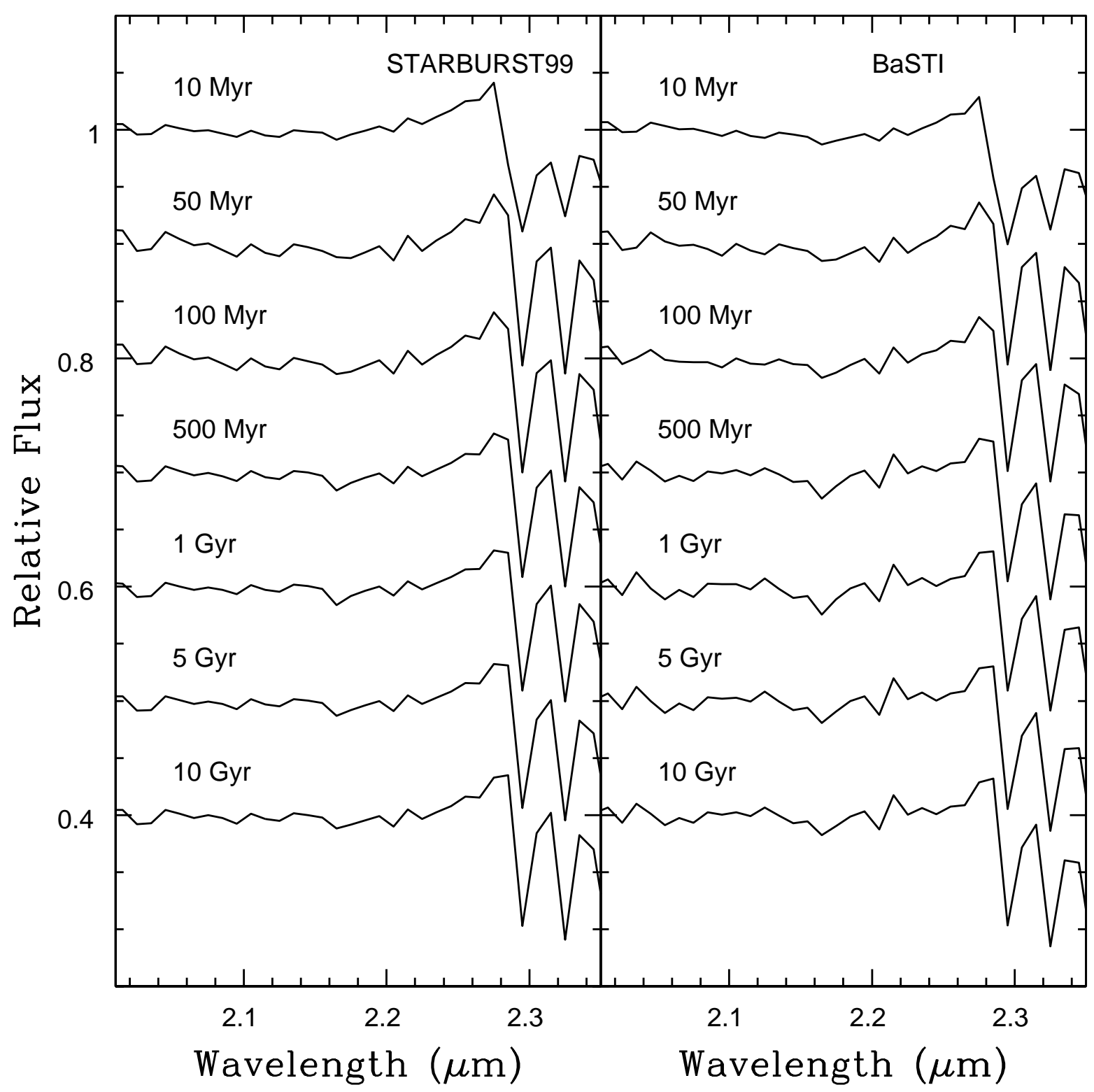

Fig. 12.- Same as Figure 11, but showing $K$-band models. The first-overtone CO bands are the most prominent absorption features in this wavelength range. The depths of the $\mathrm{CO}$ band heads differ by a few percent with age, and are deepest in the 50 and $100 \mathrm{Myr}$ models. The strengths of many features between 2.0 and $2.25 \mu \mathrm{m}$, which are heavily blended molecular and atomic transitions, also vary with age. 
makes them of potential interest as metallicity indicators.

There are age-related differences in the strengths of weaker absorption features in both spectral regions. These weaker features are blends of molecular and atomic transitions and their depths vary by a few percent for ages $\geq 30$ Myr. The peak between the $(2,0)$ and $(3,1)$ CO bands is also noticeably smaller in the 10 Myr model than in the other models.

Significant differences between the STARBURST99 and BaSTI models can be seen, with the BaSTI models having larger age-related variations and deeper absorption features. These differences are greatest in the $H$-band, and the most striking difference is associated with the Ballick-Ramsey $\mathrm{C}_{2}$ band. This feature is abscent in the STARBURST99 models, but is clearly present in the BaSTI cSFR models with ages > 500 Myr. While C stars are usually associated with systems that have sub-solar metallicities, moderate numbers of C stars might be expected in solar metallicity systems. Karakas (2014) finds that solar metallicity stars with masses between 2 and $4.5 \mathrm{M}_{\odot}$ will become $\mathrm{C}$ stars. The ratio of the number of $\mathrm{C}$ stars to the number of O-rich AGB stars of spectral-type $\mathrm{M}-\mathrm{C} / \mathrm{M}-$ in intermediate age solar-metallicity systems is $\sim 0.07$ in her models. Studies of $\mathrm{C}$ stars in the Galactic disk suggest that the lower mass limit for $\mathrm{C}$ star formation may even extend to lower values than predicted by the Karakas models (e.g. Feast, Whitelock, \& Menzies 2006), potentially increasing the possible constribution from $\mathrm{C}$ stars to the integrated NIR light.

The BaSTI cSFR models predict that $\mathrm{C}_{2}$ absorption should be present if star-forming activity has occured over a time interval of at least a few hundred Myr. These models further suggest that an exceptionally high SFR during intermediate epochs is not required to produce detectable $\mathrm{C}_{2}$ absorption. Rather, $\mathrm{C}_{2}$ might be detectable if the SFR during intermediate epochs is simply comparable to that at other epochs and the noise level in the spectrum is no larger than a few percent - if the BaSTI models are correct then $\mathrm{C}_{2}$ absorption with a depth of a few percent should be common in the spectrum of solar and sub-solar metallicity systems that have experienced extended periods of star formation during intermediate epochs. That 
$\mathrm{C}_{2}$ absorption is present in the model spectra of systems that have experienced a cSFR over a large fraction of the Hubble time is probably due to $\mathrm{C}$ stars being intrinsically brighter in the NIR than older TP-AGB stars. C stars then contribute significantly to the NIR light of composite systems even though they form only over a limited range of stellar masses.

\subsection{Comparisons with Observed Spectra}

The NGC 253 East and West spectra differ in the strengths of some emission features, but have very similar absorption features. The means of the NGC 253 East and West spectra were thus computed, and the results will be referred to as the circumIRC (cIRC) spectra. The spectra of the IRC, the cIRC, and the nucleus of NGC 7793 are compared with various models in Figures $13(H)$ and $14(K)$. The GNIRS spectra have been smoothed to match the $0.01 \mu \mathrm{m}$ resolution of the models.

\subsection{1. $N G C 7793$}

The NGC 7793 spectrum is considered first, as it is free of obvious emission features. There is mixed success in matching the NGC 7793 spectrum with the models. The absorption feature in the NGC 7793 spectrum near $1.76 \mu \mathrm{m}$ overlaps with the $\mathrm{C}_{2}$ feature in the BaSTI model spectra. However, many of the weaker features in the NGC 7793 spectra do not have obvious counterparts in the models. This might be attributed in part to binning, as the method by which binning is done can alter the appearance of low resolution spectra (e.g. Figure 6 of Davidge et al. 2015). Binning will presumably be less of a factor for very strong features, such as the depths of the first-overtone CO bands, and the CO bands in the NGC 7793 spectrum are slightly weaker than in the solar metallicity models.

That the $K$-band model spectra have deeper CO bands than in the NGC 7793 nuclear spectrum might suggest a slightly sub-solar metallicity for the nucleus of NGC 7793. This 


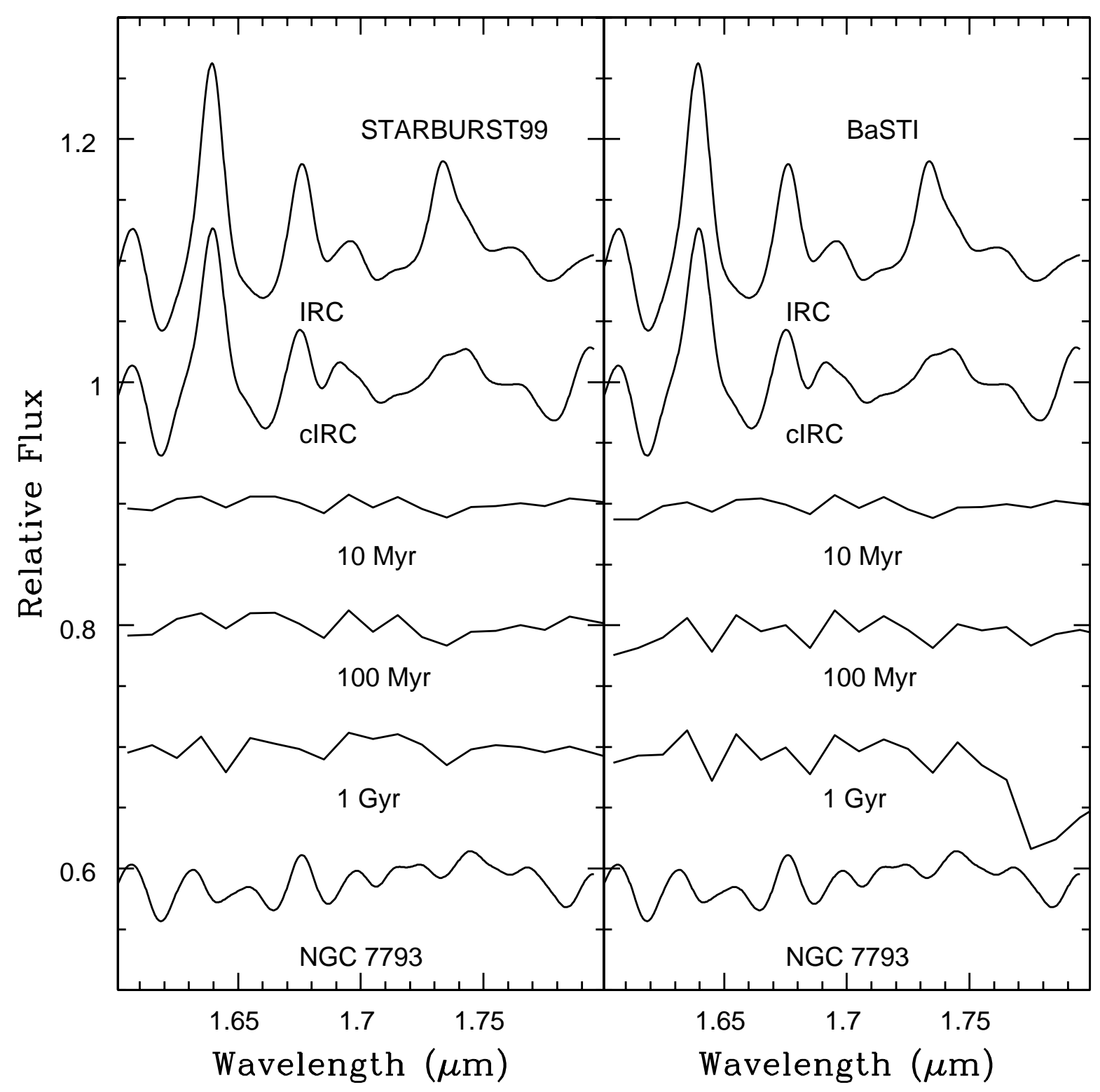

Fig. 13.- Comparisons with $H$-band models. The GNIRS spectra have been smoothed to match the resolution of the models. While strong emission lines complicate comparisons between the NGC 253 spectra and the models, this is not a significant factor for NGC 7793, although weak emission lines may become apparent after smoothing (see text). Features in the NGC 7793 spectrum tend to be stronger than predicted by the solar metallicity STARBURST99 models. There is an absorption feature at $\lambda>1.78 \mu \mathrm{m}$ in the NGC 7793 spectrum that coincides with the $\mathrm{C}_{2}$ feature in the BaSTI models, but has a smaller amplitude. A similar absorption feature is also seen at this wavelength in the NGC 253 spectra. 


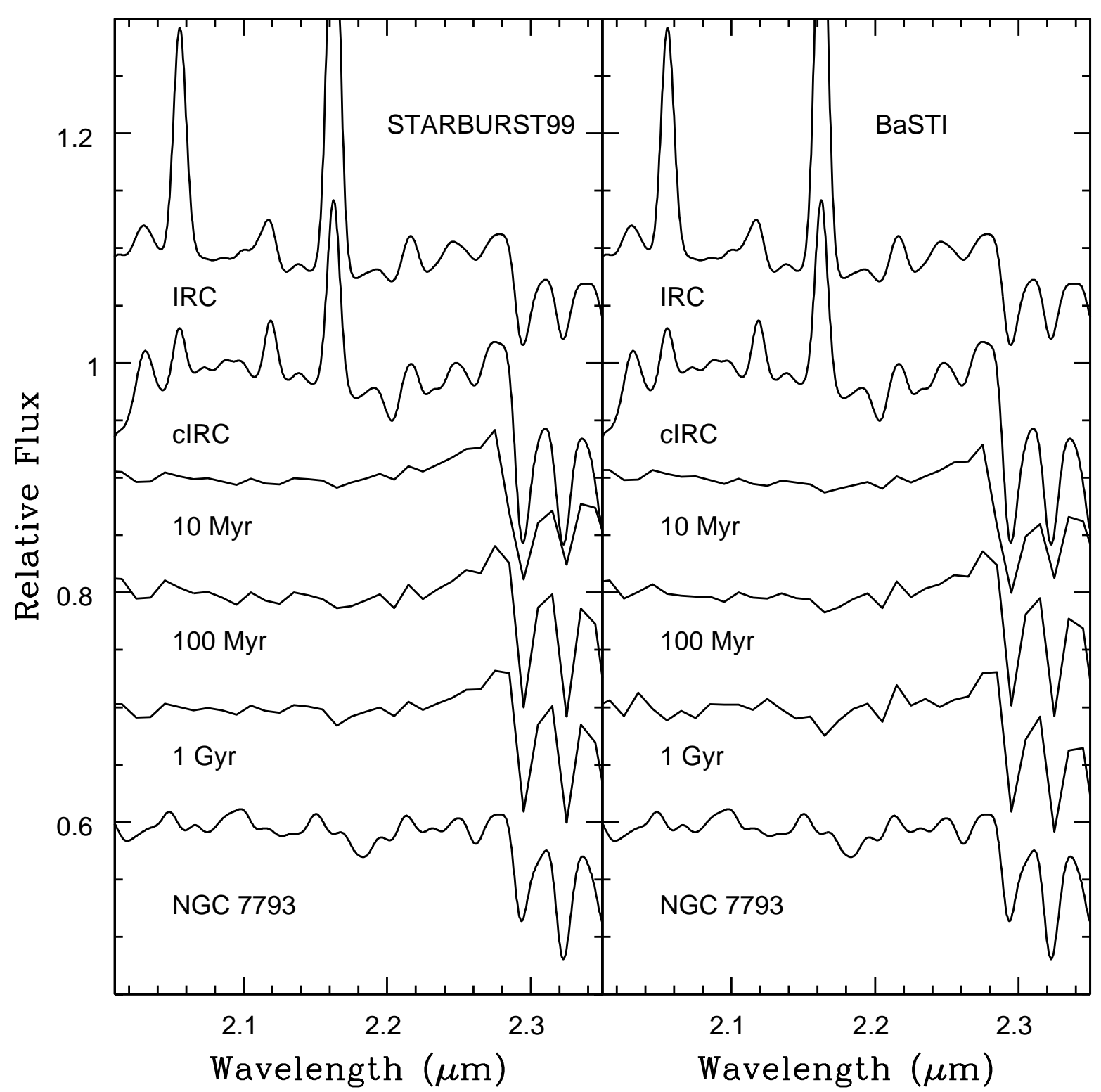

Fig. 14.- Same as Figure 13, but showing $K$-band models. The first-overtone CO bands in NGC 7793 and the IRC are slightly weaker than predicted by the solar metallicity models. As noted in Section 6.2.2, veiling by hot stellar photospheric continua may weaken the depths of absorption features in the IRC spectrum by almost $10 \%$. In contrast, the CO bands in the cIRC spectrum are deeper than in the $100 \mathrm{Myr}$ and 1 Gyr models. 
being said, Bibby \& Crowther (2010) find a central O abundance of $12+\log (\mathrm{O} / \mathrm{H})=8.6$ for NGC 7793, which is consistent with solar (e.g. Asplund et al. 2004). However, [O/Fe] has not yet been measured in NGC 7793. In addition, if the NIR light from the nucleus is dominated by stars that formed during intermediate epochs then the nucleus might have a lower luminosity-weighted metallicity than the present-day gas.

Weak line emission may become evident when the NGC 7793 spectra are smoothed, as this increases the $\mathrm{S} / \mathrm{N}$ ratio. In fact, subtle features appear in the smoothed NGC 7793 nuclear spectrum that coincide with emission features in the NGC 253 spectra. A modest population of hot young stars that power this emission may then be present in the NGC 7793 nucleus. That only a modest number of young or intermediate age stars are present near the center of NGC 7793 is perhaps not surprising. The frequency of blue nuclei among nearby disk galaxies suggests that the duty cycle for central star formation is on the order of $\sim 1$ Gyr (e.g. Davidge \& Courteau 2002; Ho et al. 1997), and the weak emission line contribution might then indicate that the nucleus of NGC 7793 is currently between largescale star-forming episodes.

\subsection{2. $N G C 253$}

The BaSTI and STARBURST99 models both underestimate the total amplitude of the $(2,0) \mathrm{CO}$ band head in the cIRC spectrum. The comparisons in Figure 14 thus suggest that the brightest RSGs in the cIRC have a metallicity that is slightly super-solar. The situation is different for the IRC, as the first overtone CO bands in that system are weaker than in the solar metallicity models. Given that the overall depths of the CO bands do not change greatly with SFH (Figure 12), and that veiling by nebular emission does not change the depths of absorption features by huge amounts (Section 4), then the difference in the CO depths between the models and the IRC spectrum might suggest that the IRC has a sub-solar metallicity. However, such a conclusion assumes that the NIR light is dominated 
by red stars.

Veiling of the CO bands could be significant if a large fraction of the NIR light originates from hot young stars, which have a near-featureless NIR spectrum. Previous NIR spectroscopic studies of the central regions of NGC 253 have concluded that it is an area of intense recent star formation (e.g. Engelbracht et al. 1998). The presence of strong HeI and $\operatorname{Br} \gamma$ emission throughout the central regions of NGC 253 indicates that hot, young stars are present in large numbers, and the areas of the IRC that have blue NIR colors in Figure 1 indicate that hot stars contribute significantly to the NIR light there.

The fraction of the mass of recently formed stars that can veil the absorption spectrum depends on the assumed age of the IRC. Consider the effect of adding a population with an age of 4 Myr (i.e. a population with massive stars that might power HeI emission) to that of the cSFR STARBURST99 models with ages of 50 Myr, 500 Myr, and 10 Gyr. These ages were selected to represent different evolutionary cases for NGC 253. The 50 Myr age explores the hypothesis that the IRC is relatively young, as might be the case if the starburst was only recently triggered. The $500 \mathrm{Myr}$ model is included to represent the case in which the IRC formed as part of a mature starburst that has been continuing for a few disk crossing times. Finally, the 10 Gyr model explores the case in which the IRC is an old structure - possibly the nucleus of NGC 253 - that has experienced periodic levels of elevated starforming activity throughout the age of the Universe, and is once again the site of renewed star-forming activity. If the absorption feature in the IRC spectrum near $1.78 \mu \mathrm{m}$ is due to $\mathrm{C}_{2}$ then this favors the $500 \mathrm{Myr}$ and 10 Gyr scenarios.

Assuming that the red stars in the IRC have solar metallicities, then the fractional contributions that a 4 Myr population must make to the total mass of the IRC to diminish the depth of the $(2,0)$ CO band in the solar metallicity model spectrum to that seen in the IRC are 70\% (50 Myr assumed age for the IRC), 30\% (500 Myr assumed age), and 10\% (10 Gyr assumed age). The fractional contribution from the 4 Myr population drops 
with increasing IRC age because the $\mathrm{M} / \mathrm{L}$ ratio in $K$ grows with increasing age, so that a smaller mass of 4 Myr stars is required to alter the spectrum of the underlying system as its age increases. Still, the assumed age of the IRC notwithstanding, the IRC is evidently being observed at a special time when it is experiencing rapid growth in stellar mass. If (1) the current level of star formation has remained constant during recent times and does not change over the near future, and (2) the stars that form in the IRC stay bound to that structure, then the times required to double the original stellar mass of the IRC are $3 \mathrm{Myr}$ (50 Myr IRC age), 9 Myr (500 Myr age), and 36 Myr (10 Gyr age).

When compared with the IRC, there is smaller fractional contamination by hot stars in the cIRC spectrum, and so the absorption features are less affected by veiling. The HeI line in the cIRC spectrum is only one-third as strong as in the IRC spectrum, while the $\mathrm{Br} \gamma$ line is roughly half as strong as in the IRC. If the light from the $4 \mathrm{Myr}$ component in the models discussed above is decreased by a factor of 3 then the $(2,0) \mathrm{CO}$ band strength is diminished by only $\sim 3 \%$, as opposed to the $7 \%$ change that is predicted for the IRC.

If the cIRC spectra sample a larger mix of stars with ages $\geq 8$ Myr than the IRC spectra then a larger fraction of the NIR light in the cIRC will come from bright RSGs. A larger fractional contribution from very young RSGs - with a resulting smaller fractional contribution from hot stars - could explain (1) why the CO bands in the East and West spectra have similar depths despite differences in the strength of $\operatorname{Br} \gamma$ emission, and (2) why the $\mathrm{CO}$ bands in the pockets of strong $\mathrm{HeI}$ and $\mathrm{Br} \gamma$ emission seen in Figures 8 and 9 are not weaker than in the surrounding areas. Based on NIR colors, Engelbracht et al. (1998) argue that there is no significant veiling of the spectrum of the center of NGC 253 by a featureless continuum, and this is more-or-less consistent with the subdued hot stellar continuum level inferred here for the cIRC region. In contrast, the F128N-F164N color data in Figure 1 clearly shows that the IRC contains regions with blue NIR colors, where veiling is expected to be significant. 
We close this section by noting that the addition of a hot stellar continuum will weaken other absorption features, including the trough at $1.78 \mu \mathrm{m}$ that might belong to $\mathrm{C}_{2}$. Adding a hot stellar continuum to the cIRC $H$-band spectrum so as to reproduce the depths of the first overtone $\mathrm{CO}$ bands in the IRC will weaken $\mathrm{C}_{2}$ absorption by $50 \%$. This is more-or-less consistent with the relative depths of the candidate $\mathrm{C}_{2}$ features in the $\mathrm{cIRC}$ and IRC spectra in Figure 13. Veiling of this size will also bring the depth of $\mathrm{C}_{2}$ absorption in the BaSTI models into better agreement with what is seen in the cIRC and IRC spectra.

\section{DISCUSSION \& SUMMARY}

The stellar content near the center of the starburst galaxy NGC 253 has been investigated using NIR IFU spectra recorded with GNIRS on GS during sub-arcsec seeing conditions. The GNIRS IFU samples a $\sim 120 \operatorname{arcsec}^{2}$ area, which corresponds to $\sim 0.03$ $\mathrm{kpc}^{2}$ at the distance of NGC 253. The IRC, which defines the photometric center of the galaxy, and several nearby sub-structures are sampled.

The analysis of integrated NIR light is at present one of the few means of examining the stellar content of the crowded central regions of NGC 253. The use of an IFU allows the spectrum of the IRC to be de-coupled from that of the surroundings, and the spatial distribution of stellar types to be mapped. It is clear that the IRC is not an SSP. As noted by Kornei \& McCrady (2009), the presence of deep CO absorption bands are indicative of RSGs, which form in systems that have an age of at least 8-10 Myr. However, the presence of $\mathrm{Br} \gamma$ and $\mathrm{HeI}$ emission indicate that IRC has been the site of highly elevated levels of star formation within the past $\sim 10 \mathrm{Myr}$, The IRC thus contains stars with an age spread of at least a few Myr. Barring a termination of star-forming activity then the age spread will increase with time. Indeed, the total stellar mass of the IRC is expected to double over time scales of a few Myr to a few tens of Myr if the pace of star formation that occured during the past 10 Myr continues unabated. 
The observations presented here indicate that the youngest stars are not well-mixed with the stars that contribute to the NIR continuum in and around the IRC. Excess $\operatorname{Br} \gamma$ emission with respect to light from the stars that dominate the NIR continuum is found in an arc along the western boundary of the IRC, while concentrated HeI emission is seen to the north of the IRC. Recent episodes of star formation have thus not been restricted to the center of the IRC, but have also occured in its outer regions.

A key issue is the age of the IRC. Is it a young - probably transient - stellar ensemble, or is it a much older structure that may be the nucleus of NGC 253? In Section 7.1 we discuss the recent SFH of the IRC, and consider its fate if it is a young structure. In Section 7.2 the possibility that the IRC is the old nucleus of NGC 253, as suggested by Gunthardt et al. (2015), is considered.

\subsection{The Recent SFH of the IRC}

That recent star formation has occured at different locations around the IRC suggests that it is likely not a 'cluster', but rather a compact star-forming complex containing distinct sub-structures. Only the sub-structures in the outer region of the system are detected with GNIRS, due to crowding in the denser inner regions. Sub-structuring on finer scales is seen in the WFC3 images in Figure 1, which have angular resolutions that are $\sim 3-4 \times$ finer than the GNIRS data.

An age dispersion of at least a few Myr is expected in young systems. There is evidence for periods of star formation in young Local Group clusters that can last up to at least a few Myr. One of the largest young systems in the Galaxy is Westerlund 1, and it has an

age dispersion of a few Myr (Kudryavtseva et al. 2012; Lim et al. 2013). A similar age spread is seen in Westerlund 3 (Bik et al. 2014). Such a short time scale for the formation of a second stellar generation in large star clusters is consistent with simulations of thermally 
stable clumps that can cool and form stars as a result of self-shielding (Palous et al. 2014).

Star formation may extend over much longer timescales than is seen in young Galactic clusters. The largest nearby star-forming complex to be surveyed in depth is 30 Doradus, and de Marchi et al. (2011) find an age dispersion of $\leq$ a few tens of Myr. Signatures of extended star formation are found in the spectra of some extragalactic clusters (e.g. Peacock et al. 2013, but see also Bastian et al. 2013). Conroy \& Spergel (2011) argue that star clusters with masses $>10^{4} \mathrm{M}_{\odot}$ can retain star-forming material for extended periods of time, although the escape velocity prior to the onset of mass segregation may provide a better predictor of gas retention than total cluster mass (Goudfrooij et al. 2014). Indeed, while there is photometric evidence for a range of stellar ages in some massive LMC clusters (e.g. Keller et al. 2012; Li et al. 2014), there are exceptions (e.g. Bastian \& Silva-Villa 2013). Multiple episodes of star formation have also been suggested as a means of explaining the chemical properties of stars in globular clusters, and CMDs indicate that many globular clusters are not SSPs (e.g. review by Gratton et al. 2012).

If the IRC has a young age then it may not be long-lived. Fall \& Chandar (2012) present evidence that the majority of clusters are disrupted very early in their lives, and the tidal environment near the center of NGC 253 may not be conducive to the long term survival of even a massive cluster. It is tempting to compare the IRC with the Arches cluster, which has an age of only a few Myr but shows signs of tidal disruption in its global mass function (Stolte et al. 2005) and radial structure (Habibi et al. 2013). However, the Arches is roughly an order of magnitude less massive than the IRC.

That only modest levels of structuring are seen in the cIRC region does not indicate that the young stars found there at the present day formed in situ as part of a diffusely distributed field population. Rather, these young stars more likely formed in clusters that are now either defunct or largely disrupted. Stars that form in the outer regions of the IRC may be susceptible to being calved off, and so these areas may also be a source of future 
stellar generations in the cIRC environment.

\subsection{Is the IRC Old?}

Gunthardt et al. (2015) argue that the IRC is the nucleus of NGC 253. Indeed, the integrated brightness and angular size of the IRC are similar to those of other galactic nuclei. A complicated SFH also does not preclude the IRC from being the nucleus, as it is not uncommon for the nuclei of late-type galaxies to host star clusters (e.g. Boker et al. 2002; Georgiev \& Boker 2014). This being said, there is a tendency for the photometric sizes

of the nuclei of late-type spirals to vary with wavelength, in the sense that most systems tend to have smaller sizes towards shorter wavelengths, suggesting a tendency for younger stars to be more centrally concentrated than the main stellar body (e.g. Georgiev \& Boker 2014). This is not the case in the IRC.

If the IRC is the nucleus of NGC 253 then a substantial age spread would be expected. Deep $\mathrm{CN}$ and $\mathrm{C}_{2}$ bands are NIR spectroscopic signatures of an underlying intermediate age population that would indicate that the IRC has survived disruption and formed stars for at least many hundreds of Myr. It is thus interesting that there is an absorption feature in the IRC spectrum near $1.78 \mu \mathrm{m}$ that may be due to $\mathrm{C}_{2}$. In Section 4 it is argued that this absorption is likely not due to DIBs, and this could be checked using higher spectralresolution observations of the center of NGC 253 in the $1.7-1.8 \mu \mathrm{m}$ wavelength interval.

The depths of the CO bands in the cIRC spectrum are consistent with a near-solar metallicity, and so the detection of C stars in significant numbers near the center of NGC 253 might be surprising. However, models of C star evolution investigated by Karakas (2014) indicate that $\mathrm{C}$ stars can form at solar and super-solar metallicities, although the results for super-solar metallicities depend critically on the Helium content. The Karakas (2014) models predict that $\mathrm{C}$ stars in solar metallicity systems have progenitor masses between 2 
and 4.5 $\mathrm{M}_{\odot}$, which corresponds to main sequence turn-off ages $\sim 0.2-1.8$ Gyr. Based on the relative lifespans of $\mathrm{C}$ stars when compared with the entire AGB phase, then the ratio of C stars to M giants predicted by Karakas (2014) for a solar metallicity system in this age range is $\sim 0.07$. If the $\mathrm{M}$ giants and $\mathrm{C}$ stars in such a system have comparable intrinsic brightnesses then a modest $\mathrm{C}_{2}$ feature would be expected in the integrated NIR spectrum.

Davidge (2010) used NIR colors to identify C star candidates throughout the disk and extraplanar regions of NGC 253, and find that C stars may be present in large numbers throughout the galaxy. Near the tip of the AGB the C star candidates contribute $\sim 20 \%$ of the $K$-band light at galactocentric radii $6-10 \mathrm{kpc}$. However, a problem when identifying C stars from broad-band photometry alone is that the threshold color that separates C stars from M giants is a function of metallicity (e.g. discussion in Boyer et al. 2013), and so metal-rich $\mathrm{M}$ giants may have colors that overlap with those of more metal-poor $\mathrm{C}$ stars. The C star contribution to the total light estimated from photometric surveys alone is thus an upper limit. MIR photometry may provide a means of distinguishing between very cool $\mathrm{M}$ giants and $\mathrm{C}$ stars, as the SED of the most evolved stars in the $3-8 \mu \mathrm{m}$ wavelength interval is sensitive to the mineralogy of the circumstellar envelope (Dell'Agli et al. 2014).

In Section 6 it was demonstrated that hot young stars likely contribute significantly to the $K$-band light from the IRC. The models used in Section 6 involve only two components - a very young SSP coupled with an idealised system that has experienced a cSFR. Still, these models suggest that if the IRC is the nucleus of NGC 253, and thus contains stars that formed over a large fraction of the age of the Universe, then very young stars account for $10 \%$ of its stellar mass. This opens the possibility that the nucleus is currently experiencing an episode of very rapid growth in terms of stellar mass. If the nucleus contains a massive black hole then the present day might also be a time during which the black hole is also accreting substantial mass. While Gunthardt et al. (2015) find no signs of nuclear activity, the orientation of NGC 253 on the sky may mean that any acccretion activity is hidden. 
It is a pleasure to thank the anonymous referee for providing a prompt and comprehensive report that greatly improved the paper. 


\section{REFERENCES}

Armand, C., Baluteau, J.-P., Joubert, M., Gry, C., \& Cox, P. 1996, A\&A, 306, 593

Asplund, M., Grevesse, N., Sauval, A. J., Allende Prieto, C., \& Kiselman, D. 2004, A\&A, 417,751

Bastian, N., \& Silva-Villa, E. 2013, MNRAS, 431, L122

Bastian, N., Cabrera-Ziri, I., Davies, B., \& Larsen, S. S. 2013, MNRAS, 436, 2852

Bertelli, G., Bressan, A., Chiosi, C., Fagotto, F., \& Nasi, E. 1994, A\&AS, 106, 275

Bibby, J. L., \& Crowther, P. A. 2010, MNRAS, 405, 2737

Bik, A., Stolte, A., Gennaro, M., et al. 2014, A\&A, 561, A12

Boker, T., Laine, S., van der Marel, R. P., Sarzi, M., Rix, H-W, Ho, L. C., \& Shields, J. C. 2002, AJ, 123, 1389

Boyer, M. L., Girardi, L., Marigo, P., et al. 2013, ApJ, 774, 83

Carson, D. J., Barth, A. J., Seth, A. C., et al. 2015, AJ, 149, 170

Chabrier, G. 2001, ApJ, 554, 1274

Conroy, C., \& Spergel, D. N., 2011, ApJ, 726, 36

Cordier, D., Pietrinferni, A., Cassisi, S., \& Salaris, M. 2007, AJ, 173, 468

Davidge, T. J. 1998, ApJ, 497, 650

Davidge, T. J. 2010, ApJ, 725, 1342

Davidge, T. J., \& Courteau, S. 2002, AJ, 123, 1438

Davidge, T. J., Andersen, D., Lardière, O., et al. 2015, ApJ, in press

Dell'Agli, F., Ventura, P., Garcia Hernandez, D. A., et al. 2014, MNRAS, 442, L38

de Marchi, G., Francesco, P., Nino, P., et al. 2011, ApJ, 739, 27 
Dubbeldam, M., Content, R., Allington-Smith, J. R., Pokrovski, S., \& Robertson, D. J. 2000, Proc. SPIE, 4008, 1181

Elias, J. H., Joyce, R. R., Ming, L., Muller, G. P., Hileman, E. A., \& George, J. R. 2006, Proc. SPIE, 6269, 4C

Engelbracht, C. W., Rieke, M. J., Rieke, G. H., Kelly, D. M., \& Achtermann, J. M. 1998, ApJ, 505, 639

Fall, S. M., \& Chandar, R. 2012, ApJ, 752, 96

Feast, M. W., Whitelock, P. A., \& Menzies, J. W. 2006, MNRAS, 369, 791

Fernandez-Ontiveros, J. A., Prieto, M. A., \& Acosta-Pulido, J. A. 2009, MNRAS, 392, L16

Geballe, T. R., Najarro, F., Figer, D. F., Schlegelmilch, B. W., \& de la Fuente, D. 2011, Nature, 479, 200

Georgiev, I. Y., \& Boker, T. 2014, MNRAS, 441, 3570

Gratton, R. G., Carretta, E., \& Bragaglia, A. 2012, ARA\&A, 20, 50

Groudfrooij, P., Girardi, L., Kozhurina-Platais, V., et al. 2014, ApJ, 797, 35

Gunthardt, G. I., Aguero, M. P., Camperi, J. A., Diaz, R. J., Gomez, P. L., Bosch, G., \& Schirmer, M. 2015, AJ, 150, 139

Habibi, M., Stolte, A., Brandner, W., Hoffmann, B., \& Motohara, K. 2013, A\&A, 556, A26

Herwig, F. 2005, ARA\&A, 43, 435

Ho, L. C., Filippenko, A. V., \& Sargent, W. L. W. 1997, ApJ, 487, 568

Hopkins, P. F., Cox, T. J., Younger, J. D., \& Hernquist, L. 2009, ApJ, 691, 1168

Iben, I., \& Truran, J. W. 1978, ApJ, 220, 980

Jarrett, T. H., Chester, T., Cutri, R., Schneider, S. E., \& Huchra, J. P. 2003, AJ, 125, 525

Karachentsev, I. D., Grebel, E. K., Sharina, M. E., et al. 2003, A\&A, 404, 93 
Karakas, A. I. 2014, MNRAS, 445, 347

Keller, S. C., Mackey, D., \& Da Costa, G. S. 2012, ApJ, 761, L5

Kornei, K. A., \& McCrady, N. 2009, ApJ, 697, 1180

Kroupa, P. 2001, MNRAS, 322, 231

Kudryavtseva, N., Brandner, W., Gennaro, M., et al. 2012, ApJ, 750, L44

Lang, M., Holley-Bockelmann, K., \& Sinha, M. 2014, ApJ, 790, L33

Leitherer, C., Schaerer, D., Goldader, J. D., et al. 1999, ApJS, 123, 3

Li, C., de Grijs, R., \& Deng, L. 2014, 784, 157

Lim, B., Chun, M-Y, Sung, H., et al. 2013, AJ, 145, 46

McQuinn, K. B. W., Skillman, E. D., Cannon, J. M., et al. 2010, ApJ, 724, 49

Muller-Sanchez, F., Gonzalez-Martin, O., Fernandez-Ontiveros, J. A., Acosta-Pulido, J. A., \& Prieto, M. A. 2010, ApJ, 716, 1166

Ott, J., Weiss, A., Henkel, C., \& Walter, F. 2005, ApJ, 629, 767

Palous, J., Wunsch, R., \& Tenorio-Tagle, G. 2014, ApJ, 792, 105

Peacock, M. B., Zepf, S. E., \& Finzell, T. 2013, ApJ, 769, 126

Pietrinferni, A., Cassisi, S., Salaris, M., \& Castelli, F. 2004, ApJ, 612, 168

Radburn-Smith, D. J., Roskar, R., Debattista, V. P., et al. 2012, ApJ, 753, 138

Rayner, J. T., Cushing, M. C., \& Vacca, W. D. 2009, ApJS, 185, 289

Reines, A. E., Nidever, D. L., Whelan, D. G., \& Johnson, K. E. 2010, ApJ, 708, 26

Rieke, G. H., Lebofsky, M. J., Thompson, R. I., Low, F. J., \& Tokunaga, A. T. 1980, ApJ, 238,24

Rosenberg, M. J. F., van der Werf, P. P., \& Isreal, F. P. 2013, A\&A, 550, A12

Soto, K. T., \& Martin, C. L. 2010, ApJ, 716, 332 
Stolte, A., Brandner, W., Grebel, E. K., Lenzen, R., \& Lagrange, A-M 2005, ApJ, 628, L113

Vazquez, G. A., \& Leitherer, C. 2005, ApJ, 621, 695

Vlajic, M., Bland-Hawthorn, J., \& Freeman, K. C. 2011, ApJ, 732, 7

Wright, E. L., Eisenhardt, P. R. M., Mainzer, A. K., et al. 2010, AJ, 140, 1868 